\title{
Functional Segregation and Development of Mouse Higher Visual Areas
}

\author{
Tomonari Murakami, ${ }^{1,2}$ - Teppei Matsui, ${ }^{1,2}$ and Kenichi Ohki ${ }^{1,2,3}$ \\ ${ }^{1}$ Department of Molecular Physiology, Graduate School of Medical Sciences, Kyushu University, Maidashi, Higashi-ku, Fukuoka 812-8582, Japan, \\ ${ }^{2}$ Department of Physiology, The University of Tokyo School of Medicine, Hongo, Bunkyo-ku, Tokyo 113-0033, Japan, and ${ }^{3}$ CREST, Japan Science and \\ Technology Agency, Tokyo 102-0083, Japan
}

Recent studies suggest that higher visual areas (HVAs) in the mouse visual cortex are segregated anatomically into two visual streams, likely analogous to the ventral and dorsal streams in primates. However, HVAs in mice have yet to be characterized functionally. Moreover, it is unknown when the functional segregation of HVAs occurs during development. Here, we investigated spatiotemporal selectivity of HVAs and their development using wide-field calcium imaging. We found that lateral HVAs in the anatomical ventral stream shared similar spatiotemporal selectivity, whereas the spatiotemporal selectivity of anterior and medial HVAs in the anatomical dorsal stream was not uniform and these areas were segregated functionally into multiple groups. This functional segregation of HVAs developed and reached an adult-like pattern $\sim 10 \mathrm{~d}$ after eye opening (EO). These results suggest, not only the functional segregation of ventral and dorsal streams, but also the presence of multiple substreams in the dorsal stream, and indicate that the functional segregation of visual streams occurs gradually after EO.

Key words: functional development; functional segregation; higher visual areas; mouse visual cortex; wide-field calcium imaging

\section{Significance Statement}

Investigation of the spatiotemporal selectivity of nine higher visual areas (HVAs) in adult and developing mice revealed that lateral HVAs belonging to the putative ventral stream shared similar spatiotemporal selectivity, whereas the spatiotemporal selectivity of anterior and medial HVAs belonging to the putative dorsal stream was not uniform and these areas were segregated functionally into multiple groups. These results suggest the presence of multiple substreams within the putative dorsal stream for visuospatial processing. Furthermore, we found that initially immature functional segregation among HVAs developed to an adult-like pattern $\sim 10 \mathrm{~d}$ after eye opening. These results provide a foundation for using mouse HVAs as a model to understand parallel processing and its developmental mechanism.

\section{Introduction}

In primates, visual information is processed through the ventral and dorsal streams, two parallel visual streams in the cerebral

\footnotetext{
Received March 17, 2017; revised July 21, 2017; accepted Aug. 5, 2017.

Author contributions: T. Murakami, T. Matsui, and K.0. designed research; T. Murakami performed research; T. Murakami analyzed data; T. Murakami, T. Matsui, and K.0. wrote the paper.

This work was supported by Brain Mapping by Integrated Neurotechnologies for Disease Studies (Brain/MINDS), Japan Agency for Medical Research and Development (AMED); Core Research for Evolutionary Science and Technology (CREST), AMED; Strategic International Research Cooperative Program (SICP), AMED; and the Japan Society for the Promotion of Sciences (JSPS) (KAKENHI Grants 25221001 and 25117004 to K.0. and Research Fellowships 201204982 and 15 H06473 to T. Matsui and 20153597 to T. Murakami). We thank T. Yoshida for building the experimental setup for eye tracking, A. Honda and Y. Son o for animal care and genotyping, and the Research Support Center, Graduate School of Medical Sciences, Kyushu, for technical support.

The authors declare no competing financial interests.

Correspondence should be addressed to either Kenichi Ohki, M.D., Ph.D., or Tomonari Murakami, Ph.D., Department of Physiology, The University of Tokyo, School of Medicine, Bunkyo-ku, Tokyo 113-0033, Japan. E-mail: kohki@m.u-tokyo.ac.jp or t-mura@m.u-tokyo.ac.jp.

DOI:10.1523/JNEUROSCI.0731-17.2017

Copyright $\odot 2017$ the authors $\quad 0270-6474 / 17 / 379424-14 \$ 15.00 / 0$
}

cortex (Ungerleider and Mishkin, 1982; Felleman and van Essen, 1991; Kravitz et al., 2011 and 2013). These consist of multiple extrastriate higher visual areas (HVAs) that share similar properties within each stream and are associated with either object recognition or spatial perception, although there is some crosstalk between these streams (Maunsell and van Essen, 1983; Lewis and van Essen, 2000; Nassi and Callaway, 2009; Markov et al., 2014). In addition, it has been proposed that the dorsal stream contains multiple substreams that contribute to different spatial functions such as spatial working memory, visually guided action, and navigation (Rizzolatti and Matelli, 2003; Kravitz et al., 2011).

Recent studies have proposed that the mouse also has divergent visual streams (Wang et al., 2012; Glickfeld et al., 2014). There are at least nine retinotopically organized HVAs surrounding the primary visual area (V1) (Wang and Bulkhalter, 2007). Anatomical studies have categorized nine HVAs into two groups based on their axonal projection patterns (Wang et al., 2011 and 2012). Of the two groups, the putative ventral stream consists of 
four lateral HVAs [lateromedial area (LM), laterointermediate area (LI), postrhinal area (POR), and posterior area $(\mathrm{P})]$ and the putative dorsal stream consists of five anterior/medial HVAs [anterolateral area $(\mathrm{AL})$, rostrolateral area $(\mathrm{RL})$, anterior area $(\mathrm{A})$, posteromedial area $(\mathrm{PM})$, and anteromedial area $(\mathrm{AM})]$. Although there is some anatomical crosstalk between the two streams as with primates, their projection patterns are distinct (Wang et al., 2012).

To determine whether the anatomically defined streams in mice process visual information in a parallel manner, it is necessary to integrate functional profiles of HVAs. Previous studies have investigated the spatiotemporal selectivity of neurons in some HVAs with two-photon imaging (Andermann et al., 2011; Marshel et al., 2011; Roth et al., 2012). In a naive analogy with primates, it would be expected that HVAs belonging to the putative ventral stream would process visual features with high spatial frequency (SPF), whereas HVAs belonging to the putative dorsal stream would process features with high temporal frequency (TF) (Desimone and Schein, 1987; Priebe et al., 2003; Miura et al., 2014). Indeed, a putative ventral area, LI, prefers high SPF and putative dorsal areas, AL, RL, and AM, prefer high TF (Marshel et al., 2011). However, inconsistent with this hypothesis, a putative dorsal area, PM, prefers high SPF (Andermann et al., 2011; Marshel et al., 2011; Roth et al., 2012). In addition, results regarding a putative ventral area, LM, are controversial. One study reported that area LM prefers high TF (Marshel et al., 2011), which is inconsistent with the view that ventral regions respond to high SPF. In contrast, other studies reported that area LM prefers middle SPF (Glickfeld et al., 2013; Matsui and Ohki, 2013). Furthermore, there are three HVAs (POR, $\mathrm{P}$, and $\mathrm{A}$ ) in which the spatiotemporal selectivity has not been investigated. Therefore, a consensus on the functional properties of all nine HVAs is still lacking.

The present study investigated the spatiotemporal selectivity of all nine HVAs using wide-field $\mathrm{Ca}^{2+}$ imaging in transgenic mice, the neurons of which expressed a genetically encoded $\mathrm{Ca}^{2+}$ indicator. The spatiotemporal selectivity of HVAs suggested that four lateral HVAs belonging to the putative ventral stream were categorized functionally into the same group, whereas anterior and medial HVAs belonging to the putative dorsal stream were segregated into multiple functional groups.

These results in adult mice raised the question of how the functional segregation among HVAs was established during development. Although it is reported that spatiotemporal selectivity in the mouse V1 gradually matures after eye opening (EO) (Hoy and Niell, 2015), such developmental analysis is rarely carried out in HVAs. Recently, it has been reported that visual response magnitude of HVAs in the putative dorsal stream gradually increases after EO (Smith et al., 2017). However, Smith et al. (2017) focused only on the developmental trajectory of response magnitude to one or two stimulus parameters; the development of spatiotemporal selectivity of HVAs has not been examined. Our questions in the present study are twofold. One is whether spatiotemporal selectivity of mouse HVAs is already mature at EO or gradually matures after EO. Another is when the visual streams of HVAs obtain adult-like divergent functions during development.

\section{Materials and Methods}

Animal preparation and surgery for in vivo wide-field $\mathrm{Ca}^{2+}$ imaging. Emx1-IRES-Cre (Jackson Labs (JAX) stock \#005628) (Gorski et al., 2002), Ai38 (JAX stock \#014538) (Zariwala et al., 2012), and Ai96 (JAX stock \#024106) (Madisen et al., 2015) mice were crossed to obtain transgenic mice in which all cortical excitatory neurons expressed GCaMP.
Adult [postnatal day 50 (P50)-P70] and developing (P15, P20, and P25) transgenic mice of either sex were prepared for in vivo wide-field $\mathrm{Ca}^{2+}$ imaging. Anesthesia was induced and maintained during surgery with $3 \%$ and $1-2 \%$ isoflurane, respectively. A custom-made metal head plate was attached to the skull with dental cement (Sun Medical). The craniotomy was made over the entire visual cortex and sealed with $1 \%$ agarose and a glass coverslip. Body temperature was maintained at $37^{\circ} \mathrm{C}$ with a heating pad. For recording under anesthesia, the animals were placed under the microscope shortly after the surgery. During anesthetized recordings, mice were sedated with chlorprothixene $(0.3-0.8 \mathrm{mg} / \mathrm{kg}$; Sigma-Aldrich) and isoflurane was reduced to $0.2-0.8 \%$ (Smith and Häusser, 2010; Marshel et al., 2011; Akerboom et al., 2012; Murakami et al., 2015, Matsui et al., 2016). All experiments were performed in accordance with the institutional animal welfare guidelines set by the Animal Care and Use Committee of Kyushu University and approved by the Ethical Committee of Kyushu University.

In vivo wide-field $\mathrm{Ca}^{2+}$ imaging. In vivo wide-field $\mathrm{Ca}^{2+}$ imaging (see Fig. 1A) was performed using a macrozoom fluorescence microscope (MVX-10; Olympus) equipped with a $2 \times$ objective [ $2 \times$ MVX Plan Apochromat Lens, numerical aperture (NA) 0.25; Olympus]. GCaMP was excited by a $100 \mathrm{~W}$ mercury lamp through a GFP mirror unit (UMGFPHQ/XL; Olympus; excitation peak: $488 \mathrm{~nm}$, emission peak: 507 $\mathrm{nm})$. Calcium signals were collected at a $5 \mathrm{~Hz}$ frame rate by using a cooled CCD camera (DS-Qi1Mc; Nikon) or an sCMOS camera (Andor Zyla 4.2; Andor Technology) controlled by NIS Elements BR (Nikon). For the recording of the entire visual cortex, a rectangular region $(5 \mathrm{~mm} \times 3.75$ $\mathrm{mm}$ ) was imaged at a resolution of $320 \times 240$ pixels and at a focus of 0.5 $\mathrm{mm}$ depth from the top of the cortical surface that first came into focus. Therefore, the focus at each cortical position was not the same over the entire visual cortex. For the recording in the awake state, the animals were housed in their cages for $18 \mathrm{~h}$ after surgery and then habituated to a rotating disc treadmill for $1 \mathrm{~h}$ before imaging experiments. We recorded the movements of the treadmill using an optical mouse. The eye movements were recorded using a CMOS camera (NS1044BU; New Electronic Technology; $10 \mathrm{~Hz}$ ) and infrared illumination (LED, $850 \mathrm{~nm}$ ) and analyzed with a custom-written program.

Visual stimulation. Visual stimuli were generated by using customwritten programs in PsychoPy (Peirce, 2007). The stimulus presentation was synchronized with the frame acquisition of images using a counter board (NI USB-6501; National Instruments). We positioned a 32-inch LCD monitor $18 \mathrm{~cm}$ from the right eye of each mouse. Drifting sinusoidal wave gratings ( $99.8 \%$ contrast) were presented in an aperture $\left(40^{\circ}\right.$ diameter). For retinotopic mapping, the grating stimuli were presented in three horizontally different positions with $40^{\circ}$ intervals. To test the spatiotemporal properties, the stimulus parameter of gratings was set by a pair of six SPF and six TF (SPF $=[0.01,0.02,0.04,0.08,0.16$, and 0.32 cpd $]$, TF $=[0.5,1.0,2.0,4.0,8.0$, and $16.0 \mathrm{~Hz}])$ for wide-field $\mathrm{Ca}^{2+}$ imaging experiments. Each stimulus started with a blank period of uniform gray (4-6 s) and was followed by $4 \mathrm{~s}$ of visual stimulation. The gratings moved in four directions per second during a session $(0,180,90$, and 270 degrees) in randomized order or in a sequential order. The spatiotemporal preferences of HVAs were consistent between sequential and randomized orders of four directions (data not shown). The stimuli were presented in a random order of 36 sets of spatiotemporal frequencies and repeated 30 times, which took $\sim 2.5-3 \mathrm{~h}$

Eye tracking. In the experiments using awake mice, we detected the pupil in the camera image to track eye movements. First, we detected the pupil area by an intensity threshold $(<3 \%$ of all pixels) and slightly dilated the detected pixels. Next, the detected pupil area was fitted by an ellipse and we tracked the center of this ellipse (Saleem et al., 2013). When the pupil moved $>10^{\circ}$ for $>10$ s during one trial (36 sets of spatiotemporal parameter were presented), we determined the trial as "a trial with large eye movements" and eliminated the trial from the analysis.

Two-photon imaging. Changes of $\mathrm{Ca}^{2+}$ fluorescence in cortical neurons were monitored using a two-photon microscope (Nikon A1MP) equipped with a mode-locked Ti:sapphire laser (MaiTai Deep See; Spectra Physics). The excitation light was focused with a $25 \times$ objective (Nikon PlanApo, NA 1.10). The average laser power delivered to the 
brain was $<20 \mathrm{~mW}$, depending on the depth of focus. GCaMP was excited at $920 \mathrm{~nm}$ and the emission was filtered at 517-567 nm. Images were obtained using Nikon NIS Elements software. A square region of the cortex $(512 \times 512$ pixels, $\sim 330 \mu \mathrm{m}$ on a side $)$ was imaged at $2 \mathrm{~Hz}$ frame rate. Images were obtained from depths of $200-300 \mu \mathrm{m}$ for experiments in layers $2 / 3$ of areas $P$ and $A$. The imaging positions of area $P$ and $A$ were identified by the wide-field $\mathrm{Ca}^{2+}$ imaging before the two-photon imaging experiment. We recorded neurons in areas $\mathrm{P}$ (four planes, $n=2$ mice) and A (six planes, $n=2$ mice). To test the spatiotemporal properties of these regions, we used the gratings with five SPF and five TF $(\mathrm{SPF}=[0.01,0.02,0.04,0.08$, and $0.16 \mathrm{cpd}], \mathrm{TF}=[0.5,1.0,2.0,4.0$, and $8.0 \mathrm{~Hz}])$. In two-photon imaging experiments, images were realigned by maximizing the correlation between frames. Regions of interest (ROIs) of individual neurons were identified manually and the ROI size was a $7 \times 7$ pixels square. ROIs of neuropil were determined as rings with $\sim 10$ $\mu \mathrm{m}$ radius and ring widths were two pixels. Time courses of individual ROIs were extracted by summing pixel values within ROIs. The time course of each trial was normalized using the fluorescent signal $(F)$ of each trial and were averaged over stimulus repetitions. As with the analysis of wide-field imaging experiments, the $5 \times 5$ response matrices of individual neurons were fitted by the 2D Gaussian fitting described above.

Data analysis. Images were analyzed with MATLAB (The MathWorks) and ImageJ. The baseline $\mathrm{F}$ of each trial was the average of all $\mathrm{Ca}^{2+}$ signals during a $1 \mathrm{~s}$ period before each stimulus onset. The visual response signal (dF) of each trial was the average of $\mathrm{Ca}^{2+}$ signals during each stimulus period. To obtain a response map, we calculated the fluorescence ratio change $(\mathrm{dF} / \mathrm{F})$ map of each trial and averaged them across trials. These maps were smoothed spatially with a Gaussian filter (size: $155 \times 155 \mu \mathrm{m}$; $\sigma: 30 \mu \mathrm{m})$. The areal borders between V1 and nine HVAs were determined by referencing the retinotopic map. During the test of spatiotemporal selectivity, we determined ROIs of HVAs as the areas showing visual responses to the gratings presented at the visual field corresponding to the middle circle in the retinotopic mapping experiment; therefore, ROIs of HVAs were located away from areal borders and other ROIs (see Fig. 2A). The response matrices of individual ROIs were extracted by averaging pixel values within ROIs corresponding to each stimulus. For the 2D Gaussian fitting (Priebe et al., 2003; Andermann et al., 2011; Glickfeld et al., 2013), $6 \times 6$ response matrices were oversampled to $60 \times$ 60 matrices. The 2D Gaussian fitting was performed as follows:

$$
\begin{array}{r}
\mathrm{R}(\mathrm{SPF}, \mathrm{TF})=A \exp \left(\frac{-\left(\log _{2}(S P F)-\log _{2}\left(P_{S P F}\right)\right)^{2}}{2 \sigma_{S P F}^{2}}\right) \\
\exp \left(\frac{-\left(\log _{2}(T F)-\log _{2}\left(P_{T F}(S P F)\right)\right)^{2}}{2 \sigma_{T F}^{2}}\right)
\end{array}
$$

where $A$ is the maximum response of ROI, $P_{S P F}$ and $P_{T F}$ are the preferred SPF and TF, respectively, and $\sigma_{S P F}$ and $\sigma_{T F}$ are the tuning widths of SPF and TF, respectively. The dependence of temporal preference on SPF is captured by a power-law exponent $\zeta$, such that:

$$
\log _{2}\left(P_{T F}(S P F)\right)=\zeta\left(\log _{2}(S P F)-\log _{2}\left(P_{S P F}\right)\right)+\log _{2}\left(P_{T F}\right)
$$

We quantified the correlation coefficients between response matrices before and after 2D Gaussian fitting and excluded the low correlation data $(r<0.5)$. The discriminant analysis was performed to compare the functional properties of HVAs using College Analysis Version 6.1. The Mahalanobis distances of functional properties among HVAs were calculated using all data from individual mice.

Statistical analyses. One-way ANOVA was performed to compare the preferred SPF and TF between multiple HVAs, followed by Tukey's honest significant difference (HSD) test. For this analysis, the sample size $(n)$ was defined as the number of animals. An $F$ test with the Bonferroni correction applied was performed to test whether the Mahalanobis distances of functional properties among HVAs were separated significantly. One-way ANOVA and Tukey's HSD test were performed to compare preferred SPF or TF with the 2D correlations and the inter-areal Mahalanobis distances between developing and adult mice. For these analyses, the sample size $(n)$ was defined as the number of areal pairs. The preferred SPF and TF were transformed logarithmically for these comparisons. Throughout the study, $p<0.05$ was considered statistically significant.

\section{Results}

\section{Functional identification of areal positions of V1 and nine HVAs}

We performed wide-field $\mathrm{Ca}^{2+}$ imaging (Fig. $1 A$ ) with transgenic mice expressing a genetically encoded $\mathrm{Ca}^{2+}$ indicator, GCaMP3 (Tian et al., 2009) or GCaMP6s (Chen et al., 2013), mostly in excitatory neurons (Murakami et al., 2015). In mice, anatomical tracings have identified nine retinotopically organized HVAs, each of which is connected directly with V1 (Wang and Bulkhalter, 2007). These HVAs exhibited visual responses to grating stimuli ( $40^{\circ}$ diameter, smooth edges; Fig. $\left.1 B\right)$. When responses to stimuli presented at different retinotopic positions were overlaid in different colors, V1 and HVAs exhibited clear retinotopic organization (Fig. 1B) (Andermann et al., 2011). However, two HVAs, A and P, were obscure in the retinotopic map. The positions of these two HVAs were determined using appropriate stimulus conditions as described below.

\section{Functional classification of nine HVAs by their spatiotemporal selectivity}

To investigate the spatiotemporal response selectivity in HVAs, we used grating stimuli with various spatiotemporal frequencies. We varied SPF and TF of grating stimuli in the range of 0.01-0.32 cycles per degree (cpd) and $0.5-16.0 \mathrm{~Hz}$, respectively, resulting in a total of 36 grating stimuli (see Materials and Methods). Figure $1 C$ shows examples of the visual responses to three grating stimuli. When gratings with high SPF and low TF were presented, strong visual responses were observed in lateral and medial HVAs, such as areas POR, P, and PM (Fig. 1C, left). In contrast, anterior HVAs, such as areas RL and A, exhibited strong responses to gratings with low SPF and high TF (Fig. $1 C$, right). We determined the areal positions of two HVAs, A and P, using grating stimuli with appropriate parameters that evoked reliable visual responses in these HVAs (Fig. 1D). The positions of areas A and $\mathrm{P}$ were also confirmed by retinotopic mapping using gratings with high SPF and low TF or low SPF and high TF in other animals (Fig. 2). The gratings with high SPF and low TF or low SPF and high TF evoked clear retinotopic structures in lateral or anterior HVAs, respectively. By referring to the positions of these areas and previous anatomical reports (Wang and Bulkhalter, 2007; Wang et al., 2011, 2012), we considered a responsive area located posterior to areas LI and POR as area P (Fig. 2B,C), whereas we considered an area located anterior to areas $\mathrm{AL}$ and $\mathrm{RL}$ as area $\mathrm{A}$ (Fig. $2 D, E$ ). In some animals, we observed a hotspot caudal and medial to area $\mathrm{P}$ (Fig. $2 A$, middle). There is a possibility that this may correspond to an area that has been described in Garrett et al. (2014) to be located posterior to areas V1 and P.

Using the 36 grating stimulus conditions, we investigated quantitatively the spatiotemporal selectivity of nine HVAs, including three previously uncharacterized areas (i.e., POR, $\mathrm{P}$, and A). Figure $3 A$ shows examples of visual response profiles to 36 grating stimuli in V1 and two HVAs, POR and RL. Individual squares represent responses in the ROI within each area. These two HVAs exhibited a marked dissociation of response profiles. Area POR strongly responded to gratings with high SPF and low $\mathrm{TF}$, whereas area RL strongly responded to those with low SPF and high TF (Fig. 3A). To compare the functional properties of HVAs, we computed response matrices of V1 and nine HVAs in each mouse (see Materials and Methods). Figure $3 B$ shows the 
A

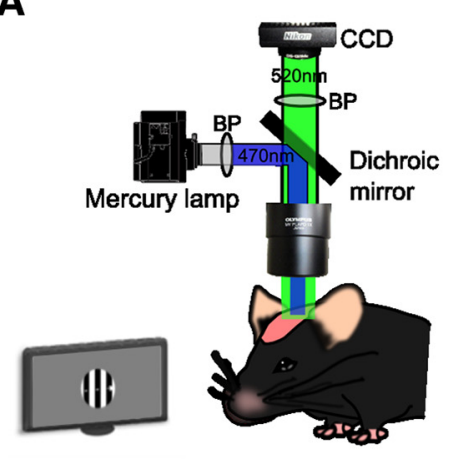

C

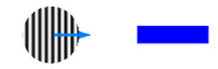

(SPF $0.16 \mathrm{cpd}-$ TF $1.0 \mathrm{~Hz}$ )
B

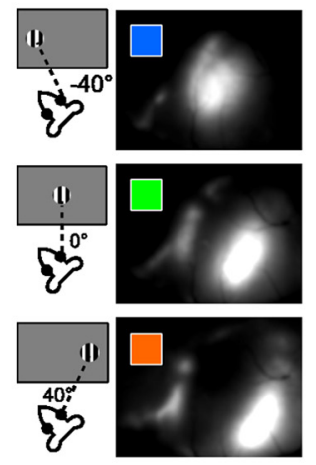

1\|\|

(SPF $0.04 \mathrm{cpd}-$ TF $2.0 \mathrm{~Hz}$ )
Retinotopic map

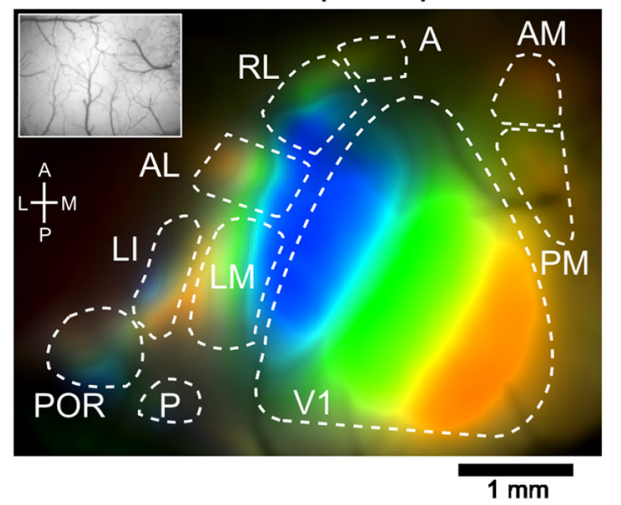

117

(SPF $0.02 \mathrm{cpd}-\mathrm{TF} 8.0 \mathrm{~Hz}$ )
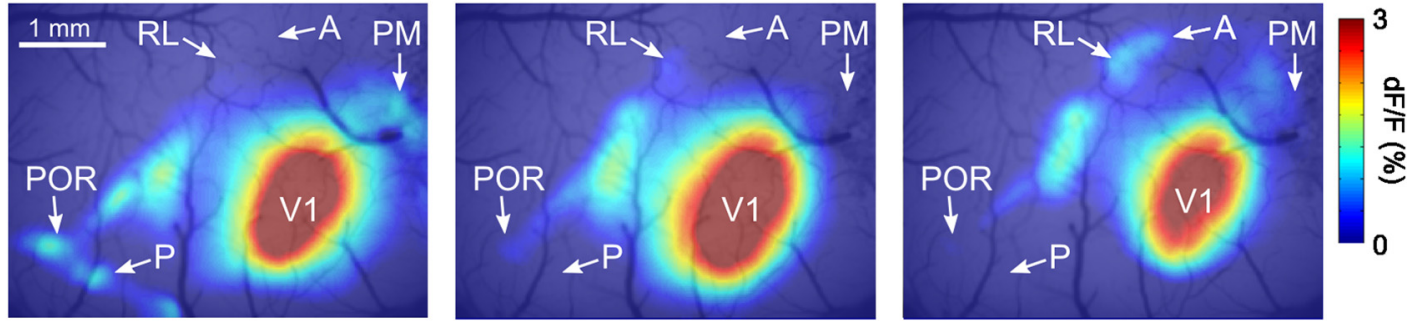

D
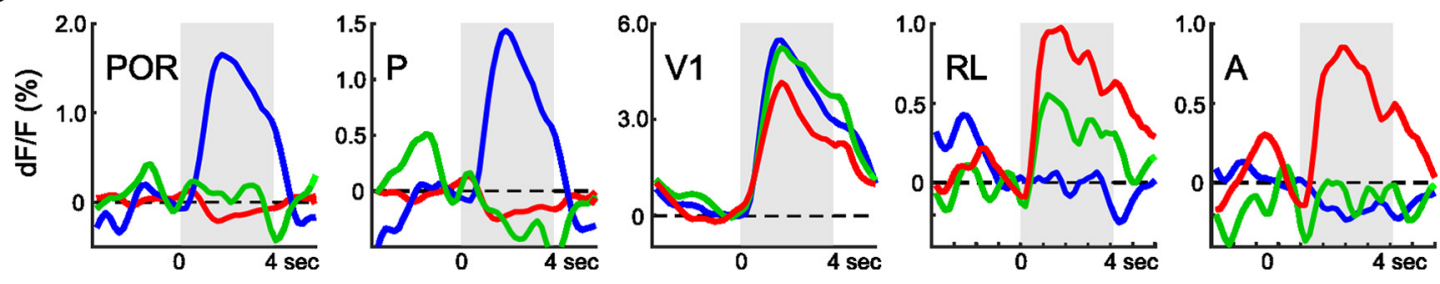

Figure 1. Determination of areal position using retinotopic mapping and the visual response to grating stimuli for various spatiotemporal frequencies. $A$, Experimental setup of wide-field $C^{2}{ }^{2+}$ imaging. $\boldsymbol{B}$, Retinotopic structure of V1 and HVAs in the entire visual cortex. Left, Maps of the calcium visual responses to grating stimuli ( $40^{\circ}$ diameter, smooth edges) that were presented at different horizontal positions on the monitor with $40^{\circ}$ intervals. Right, Merged map of the three response maps on the left showing retinotopic structures of V1 and HVAs. The inset is a region of interest in the imaging experiment. C, Examples of the responses to grating stimuli with three different spatiotemporal parameters. Grating stimuli with high SPF and low TF evoked response in lateral and medial HVAs (left), whereas stimuli with low SPF and low TF evoked response in anterior HVAs (right). D, Average time courses of calcium responses in V1 and four HVAs (POR, P, RL, and A). The line colors correspond to the three stimulus parameters in the top panels. These response maps and time courses show the visual responses and areal positions of $P$ and $A$, which are obscured in the retinotopic map in $\boldsymbol{B}$.

response matrices of $\mathrm{V} 1$ and nine HVAs averaged across seven mice (colored matrices in Fig. $3 B$ ). We fit the response matrices with a $2 \mathrm{D}$ Gaussian functions (gray-scale images in Fig. $3 B$ ) and defined the preferred SPF and TF as the peak points of the fitted Gaussians. The accuracy of the Gaussian fit was verified by computing the correlation between the non-fitted and fitted response matrices (Fig. 3C) and we excluded low correlation data $(r<0.5)$ indicating poor Gaussian fit from the following analysis ( 2 matrices out of 70 were excluded). Figure $3 D$ shows the distribution of preferred SPF and TF for nine HVAs ( $n=7$ mice). One-way ANOVA revealed the main effects of both SPF and TF across nine HVAs $\left(\right.$ SPF: $F_{(8,52)}=10.78, p<8.60 \times 10^{-9}$; TF: $F_{(8,52)}=33.72$, $p<10^{-18}$ ). Three lateral HVAs (LI, POR, and P) and area PM preferred gratings with high SPF and low TF (Fig. $3 B, D$ ). The preferred SPF in areas LI, POR, P, and PM were significantly higher than that in areas RL and A (Fig. 3D, $p<0.05$, post hoc Tukey's HSD test). In contrast, two anterior HVAs, RL and A, preferred gratings with low SPF and high TF. The preferred TF in areas RL and A were significantly higher than that in areas LM, LI, POR, P, and PM (Fig. 3D, $p<0.05$, post hoc Tukey's HSD test).
Among nine HVAs, areas LM and AL had intermediate properties (Fig. $3 B, D$ ). Consistent with a previous report, area AL was tuned to slightly higher TF than area LM (Andermann et al., 2011). Area AM was unique and had a preference for high SPF and high TF. The preferred SPF in area AM was significantly higher than that of areas RL and A; in addition, the preferred TF was higher than that of areas LM, LI, POR, P, and PM (Fig. 3D, $p<0.05$, post hoc Tukey's HSD test). Therefore, despite massive anatomical crosstalk (Wang et al., 2012), nine HVAs exhibited heterogeneous spatiotemporal selectivity.

Because two HVAs, A and P, only showed reliable visual responses to a subset of grating stimuli (Fig. 1D), we confirmed whether the visual responses in areas $\mathrm{P}$ and $\mathrm{A}$ observed by the wide-field imaging reflected the activities of neurons in these areas using two-photon imaging (Fig. 4). The neurons in these areas showed strong responses to gratings with particular parameters (Fig. $4 A-E$ ) and the spatiotemporal preferences in these areas were similar between the wide-field and two-photon data (Fig. $4 F, G$ ). Signal amplitudes of the neuropils were negligible compared with those of neurons (Fig. $4 B, D$ ), indicating that the 
A

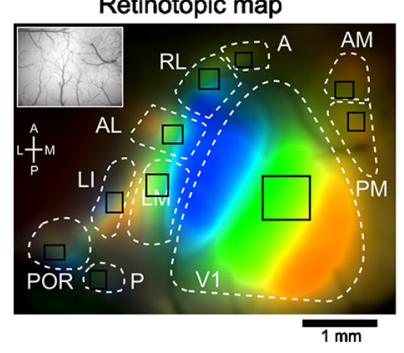

B

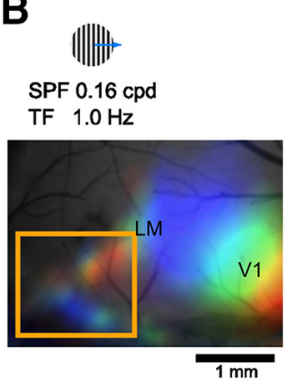

C

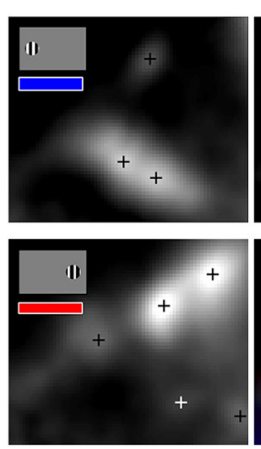

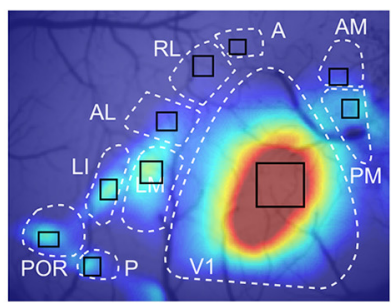

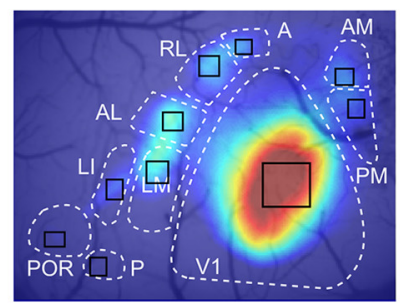

D

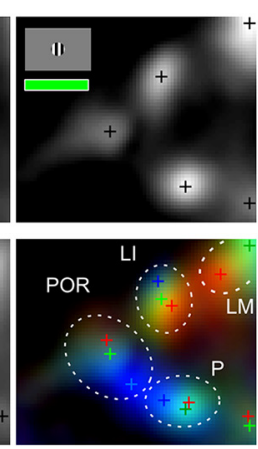

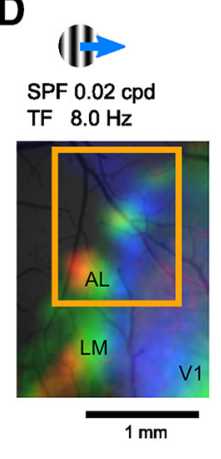

E

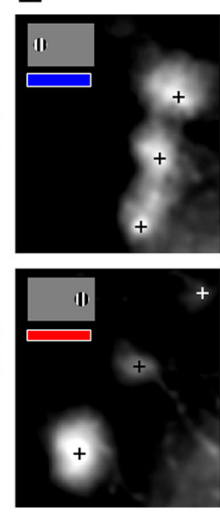

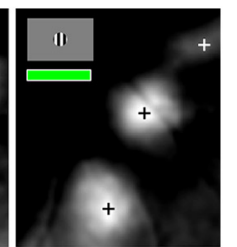

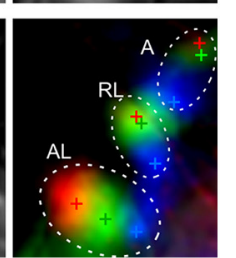

Figure 2. Retinotopic mapping using gratings with low SPF and high TF or high SPF and low TF. $\boldsymbol{A}$, Example showing ROls of HVAs. ROIs are overlaid on retinotopic map (left) and visual response maps by gratings with low SPF and high TF (middle) or high SPF and low TF (right), which are shown in Figure 1, B and C. B, C, Retinotopic structures of anterior HVAs mapped by gratings with high SPF and low TF $(\boldsymbol{B})$ or low SPF and high TF (C). D, E, Details of the retinotopic structures in the boxed regions in $\boldsymbol{B}$ and $\boldsymbol{C}$. Monochrome panels show calcium visual responses to gratings that were presented at three different positions. Cross marks represent peaks of the calcium responses. Color-merged maps of these three maps show clear retinotopic structures in lateral ( $\boldsymbol{D})$ and anterior $(\boldsymbol{E})$ HVAs.

visual responses observed by the wide-field imaging were mostly from cell bodies.

To determine whether nine HVAs were classified into functionally distinct groups, we quantified the similarities in functional properties of HVAs by a discriminant analysis calculating the Mahalanobis distances of the spatiotemporal selectivity among HVAs (Fig. 3E; see Materials and Methods). A short Mahalanobis distance between two HVAs indicates similar functional properties. The inter-areal distances among lateral HVAs belonging to the putative ventral stream (LM, LI, POR, and P) (Wang et al., 2012) were very short and significantly separated from areas AL, AM, RL, and A in the putative dorsal stream (Fig. $3 E, p<0.0014, F$ test with Bonferroni correction), showing that areas LM, LI, POR, and P are categorized functionally into the same group, as with their anatomical profiles. In contrast, within the putative dorsal stream, anterior and medial HVAs (AL, PM, AM, RL, and A) were significantly separated from each other, with the exception of areas RL and A. When we performed the same analysis using response matrices before Gaussian fit, we obtained consistent results (Fig. $3 F, G$ ). Furthermore, we confirmed that the functional segregation of spatiotemporal preference among HVAs was consistent between the awake and anesthetized mice (Fig. 5).

Together, these results indicate that lateral HVAs in the putative ventral stream form a functional group processing high SPF and low TF stimuli, whereas anterior and medial HVAs in the putative dorsal stream are segregated functionally into multiple groups: The first group (RL and A) processes low SPF and high TF stimuli; the second group (AL) prefers relatively low SPF and low TF stimuli; the third group (PM) processes high SPF and low TF stimuli; and the fourth group (AM) has a preference for high SPF and high TF stimuli.

\section{Spatiotemporal selectivity of HVAs in developing mice}

Using the same protocol as the previous experiment, we were able to study juvenile mice and explore the development of the functional segregation of HVAs. To investigate the timing of the functional dissociation of HVAs during development, we recorded the visual response in developing mice at three time points, P15, P20, and P25 (Fig. 6A-C). Developing mice open their eyes at $\sim \mathrm{P} 14$; here, we describe P15 as $1 \mathrm{~d}$ after EO (EO1). We found that HVAs were visually responsive with well defined retinotopic maps at EO1, allowing us to examine spatiotemporal selectivity from this time point. However, we note that responses in areas A and P were unclear at EO1 and these areas were excluded from subsequent analyses of EO1.

We analyzed the development of spatiotemporal selectivity in individual HVAs using the same set of gratings as used in adult experiments. Figure 6, $D-F$, shows the plots of preferred TF and SPF in each HVA at the three time points. Some functional differences of spatiotemporal selectivity, such as between areas PM and RL, were apparent in the average response matrices (Fig. 6A). One-way ANOVA of spatiotemporal selectivity at EO1 $(n=5$ mice; 4 matrices out of 40 were excluded based on the correlation between before and after Gaussian fit) revealed a significant main effect on the preferred TF among HVAs (Fig. $6 D, F_{(6,24)}=7.2$, $p<0.0002)$. Although the post hoc test revealed that there were some significantly different areal pairs for the preferred TF (Fig. $6 D, p<0.05$, post hoc Tukey's HSD test), there was no main effect on SPF preference (one-way ANOVA, $F_{(6,24)}=1.85, p=0.13$ ). 
A

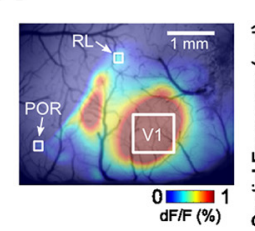

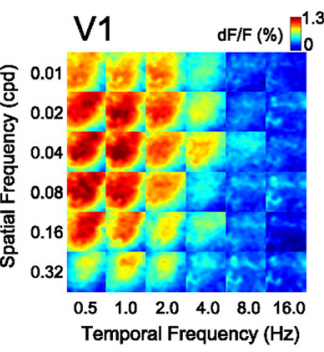

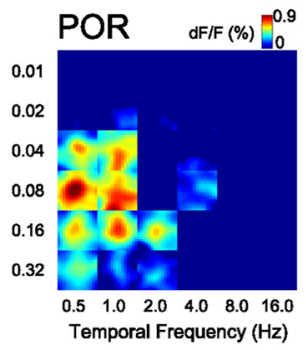

C Correlation Coefficients of 2D Gaussian fitting

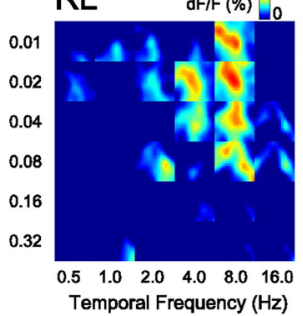

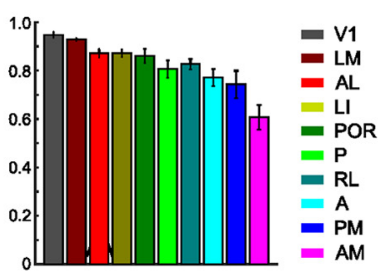

B Adult (postnatal day $60 \sim 90, \mathrm{n}=7$ mice)

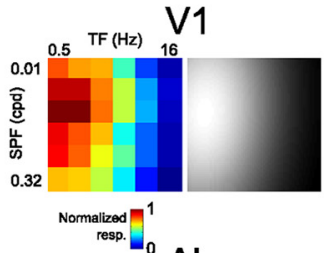

$A L$

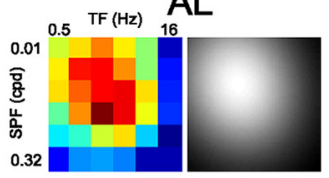

LM

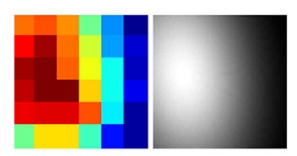

RL

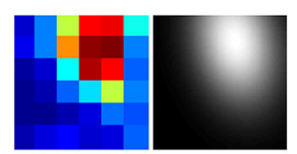

LI

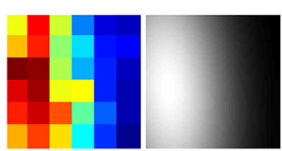

A

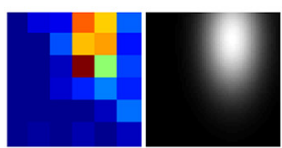

POR

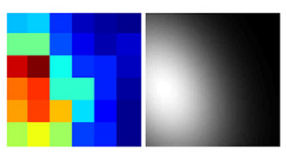

PM

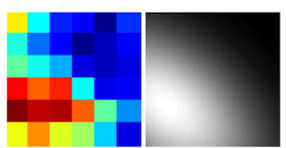

P

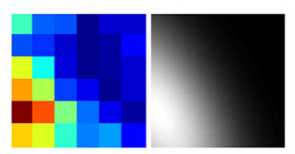

AM

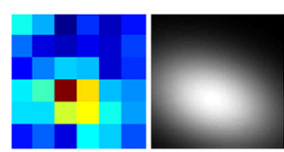

D

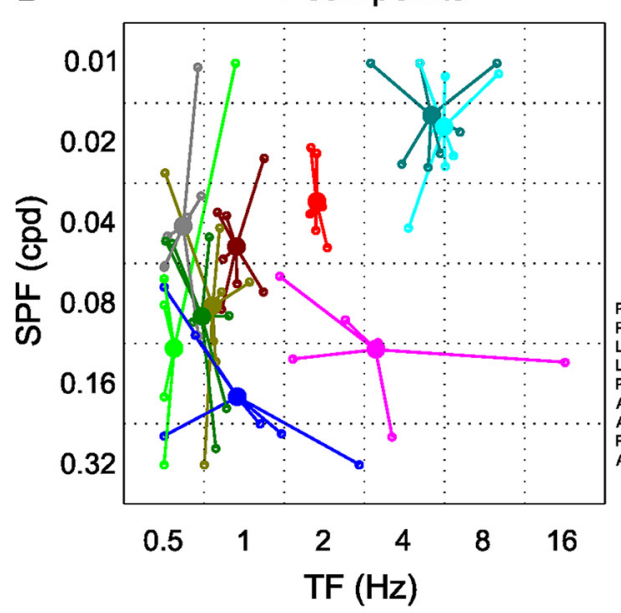

Peak points

F
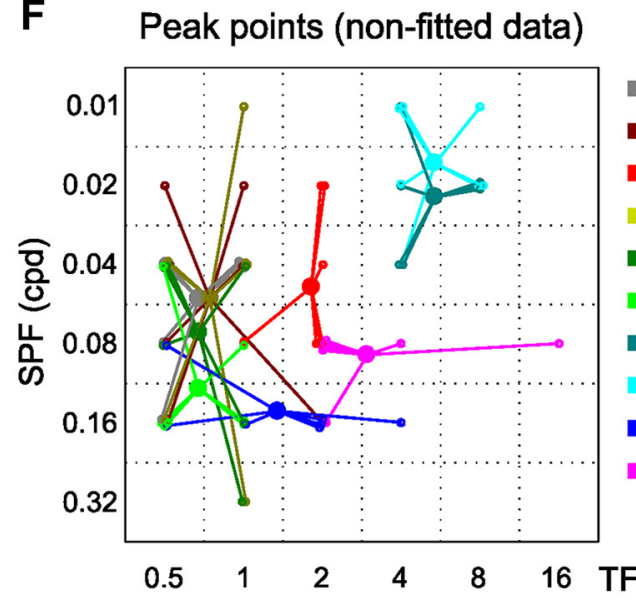

V1

- LM AM $=P$

- $\mathrm{AL}=\mathrm{LI}=\mathrm{RL}$

- PM POR A

\section{One-way ANOVA \&} post hoc HSD test

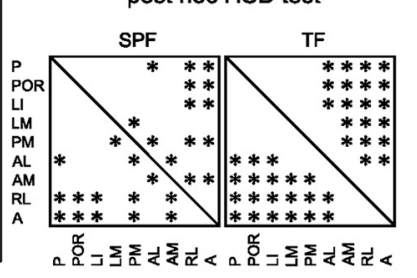

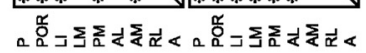

E

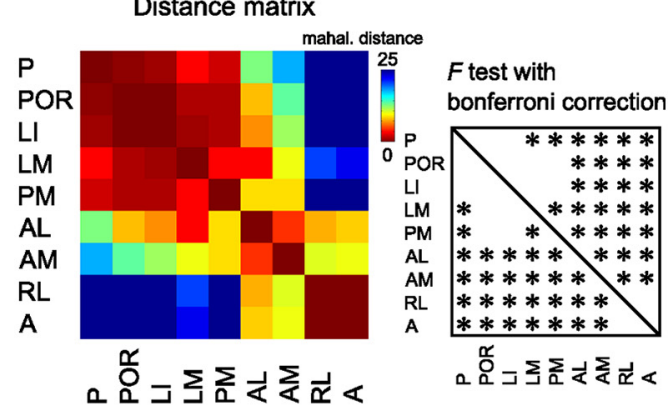

Figure 3. Functional classification of HVAs by a discriminant analysis performed on the spatiotemporal selectivity. $A$, Examples of the calcium visual responses to the 36 gratings in areas $V 1$, POR, and RL. Left, ROIs in each area. Areas POR and RL exhibited distinctly different preferences, such that area POR was strongly responsive to high SPF and low TF stimuli, whereas area RL was responsive to low SPF and high TF stimuli. B, Response matrices of V1 and nine HVAs fitted with 2D Gaussian fitting in adult mice. The left color-coded matrix in each area is the averaged (Figure legend continues.) 
A

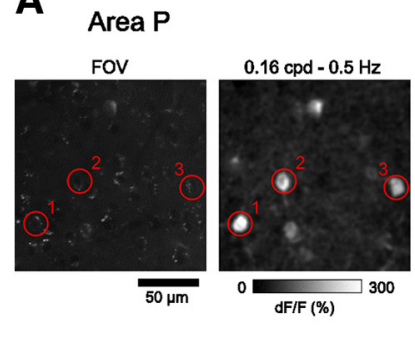

C Area A

Fov
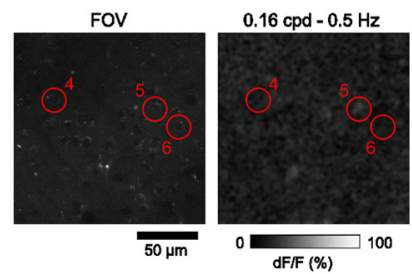

E Peak points of

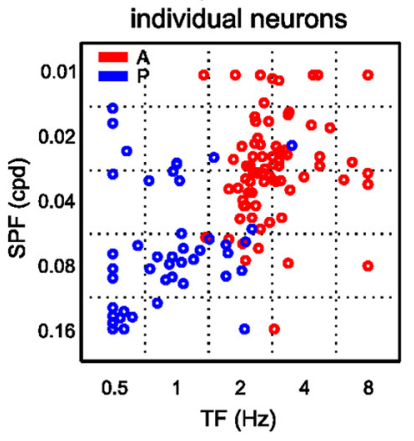

B
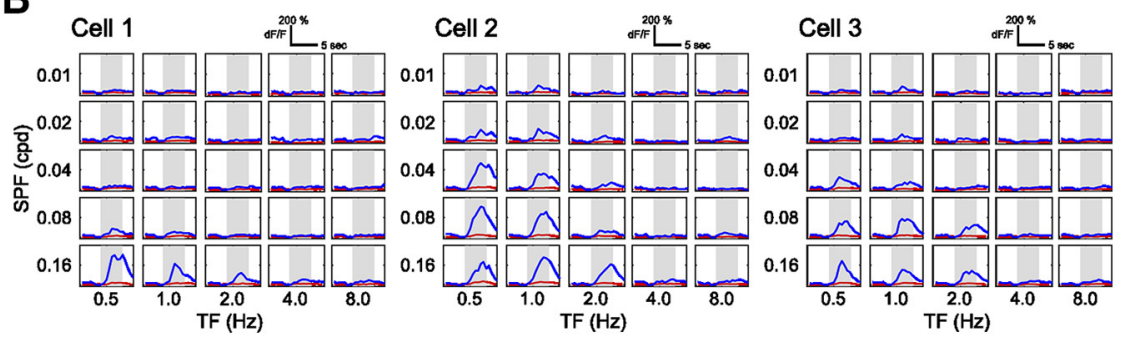

D

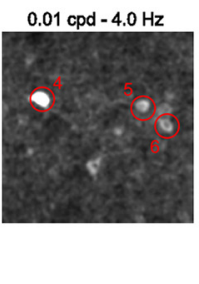

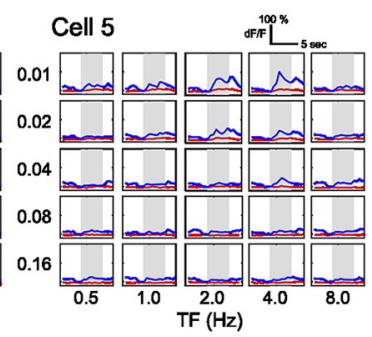

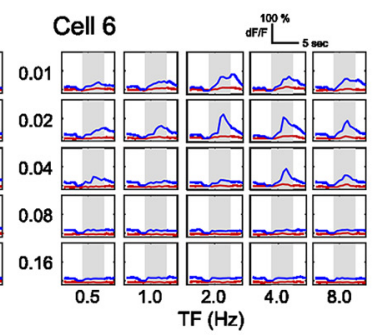

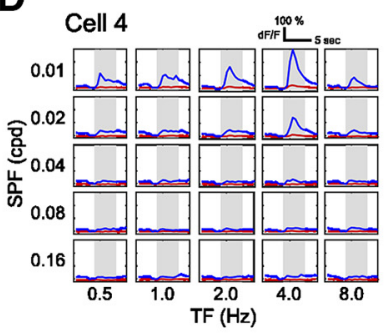

F Average response matrix of all neurons

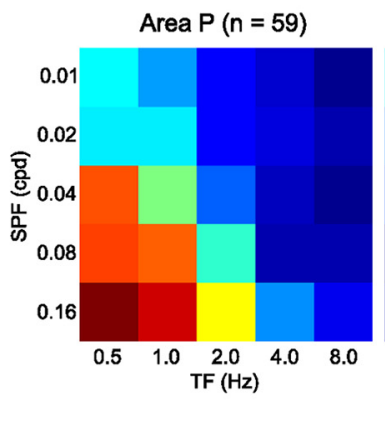

Area $A(n=77)$

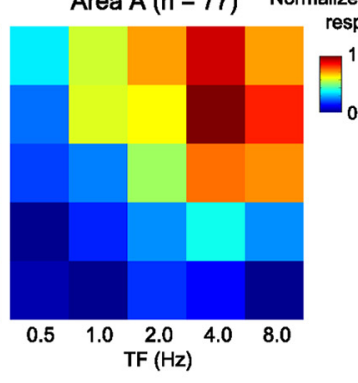

G

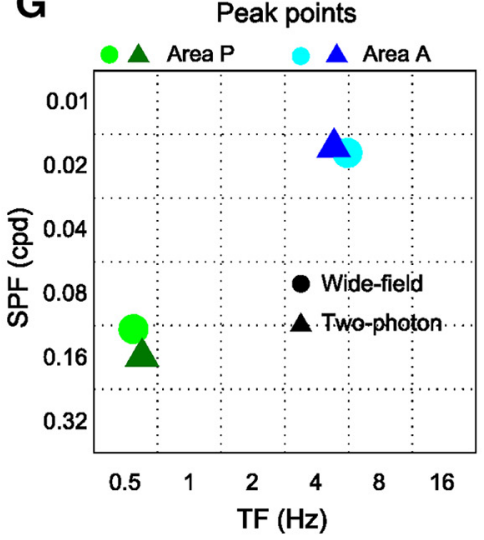

Figure 4. Spatiotemporal preferences of individual neurons in areas $P$ and A. $A$, Examples of the neural responses in area $P$ to grating stimuli with two different spatiotemporal parameters. Left, Extracted FOV. Grating stimuli with high SPF and low TF evoked calcium responses of neurons (middle), whereas stimuli with low SPF and high TF did not (right). $\boldsymbol{B}$, Averaged time courses of representative neurons marked in $\boldsymbol{A}$. These neurons preferred gratings with high SPF and low TF. Gray shading indicates the stimulus periods. The red lines indicate the signals of neuropil around neurons. Neuropil signals were negligible compared with those of neurons. $\boldsymbol{C}, \boldsymbol{D}$, Examples of the neural responses in area A to grating stimuli with two different spatiotemporal parameters. The neurons in the FOV showed the strongest responses to grating stimuli with low SPF and high TF. E, Peak points of the fitted response matrices of individual neurons. Most of neurons in areas $P$ and A preferred the gratings with high SPF and low TF and those with low SPF and high TF, respectively. $F$, Average response matrices of all neurons in areas $P$ and $A(n=59$ and 77 neurons in areas $P$ and $A$ from two mice, respectively). The matrices were normalized from 0 to 1 , corresponding to minimum and maximum responses. $G$, Peak points of the fitted response matrices of areas $P$ and $A$ in two-photon and wide-field imaging experiments. The circles are peaks of the average response of all neurons in two-photon imaging $(\boldsymbol{F})$, whereas the triangles show the averaged peaks of all mice in wide-field imaging (the same data shown in Fig. 3D).

(Figure legend continued.) response matrices before the Gaussian fitting. C, Correlations of response matrices between before and after Gaussian fit. The correlation coefficients were high across all the visual areas. $\boldsymbol{D}$, Peak points of the fitted response matrix in adult mice $(n=7$ mice). Small open circles indicate the individual data. Large filled circles are averages of the open circles. Areas LI, POR, P, and PM preferred high SPF and low TF stimuli, whereas areas RL and A preferred low SPF and high TF stimuli. Among nine HVAs, areas LM and AL had intermediate properties. Area AM had a preference for high SPF and high TF stimuli. The differences in spatiotemporal selectivity between HVAs are significant (one-way ANOVA, SPF: $F_{(8,52)}=10.78$, $p<8.60 \times 10^{-9}$; TF: $F_{(8,52)}=33.72, p<10^{-18}$; post hoc Tukey's HSD test, $\left.p<0.05\right) . \boldsymbol{E}$, Inter-areal distance matrix representing the Mahalanobis distances of functional properties among HVAs. Areas $L M, L I, P O R, P$, and PM in the putative ventral stream were significantly separated from areas AL, AM, RL, and A ( $p<0.0014$, F test with Bonferroni correction). Within the putative dorsal stream, areas AL, PM, AM, RL, and A were not functionally uniform. $F$, Peak points of the response matrices before Gaussian fit in adult mice. Small open circles indicate the individual data. Large circles are averages of individual data. $\mathbf{G}$, Inter-areal distance matrix representing the Mahalanobis distances of the nonfitted data among HVAs.
Furthermore, the response preferences of HVAs, especially in areas LI, POR, and AM, were quite variable across individual animals. These results indicate that, although HVAs are visually responsive, functional segregation of HVAs is less obvious at EO1.

At P20 ( $n=7$ mice; 7 matrices out of 70 were excluded), areas $\mathrm{A}$ and $\mathrm{P}$ became visually responsive to grating stimuli with appropriate parameters. When we compared the preferred TF across nine HVAs, areas RL and A showed a preference for significantly higher TF than areas LI and POR (Fig. 6E, one-way ANOVA, $F_{(8,46)}=6.45, p<1.32 \times 10^{-5}$; post hoc Tukey's HSD test, $p<0.05)$. However, there were fewer significant differences in the preferred TF at P2 0 compared with adults ( 8 areal pairs out of 36 at P20 and 20 areal pairs out of 36 in adults; Fig. $6 E, G)$. Similarly, the main effect in the SPF preference was significant among HVAs (one-way ANOVA, $F_{(8,46)}=5.27, p<0.0001$ ), but the number of significant differences in P20 was fewer than that in adults to adults ( 8 areal pairs out of 36 at P20 and 14 areal pairs 
A

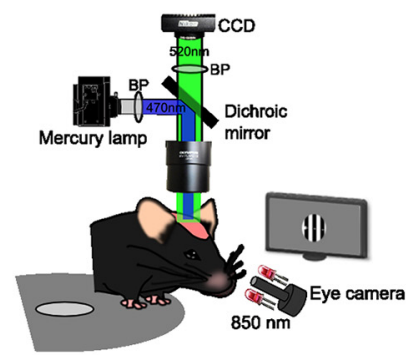

C

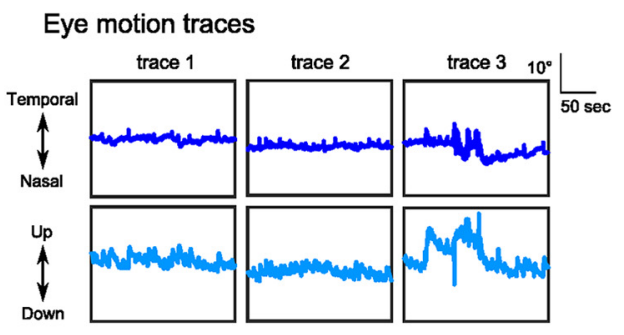

Locomotion trace

B
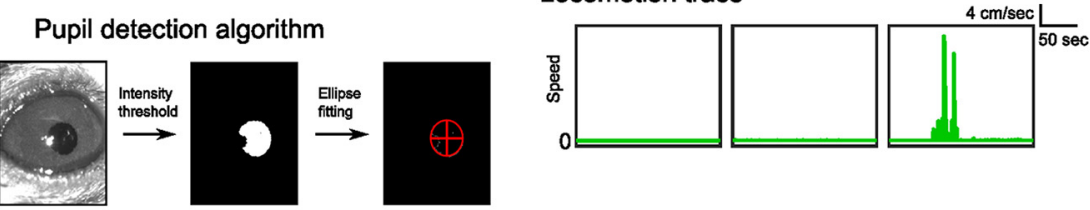

E

Awake state

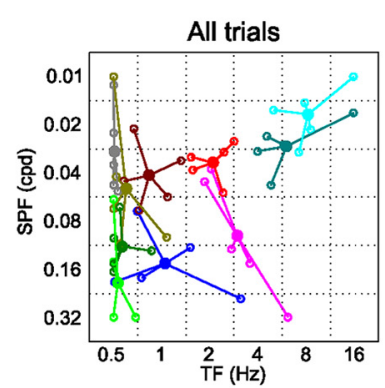

Trials without large

eye movements

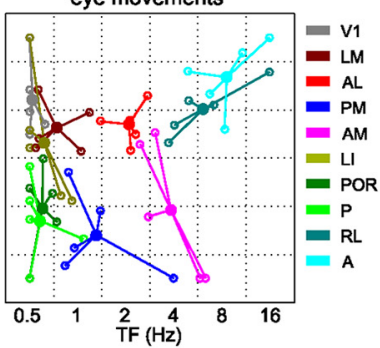

F

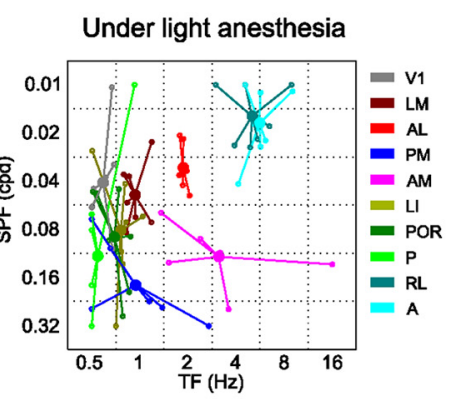

D
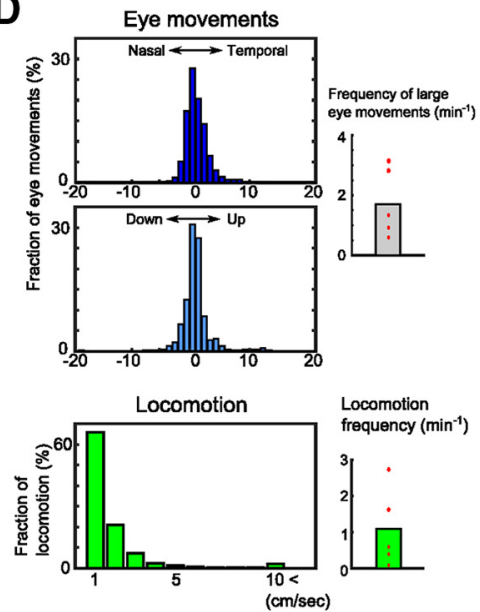

G Mahalanobis distance

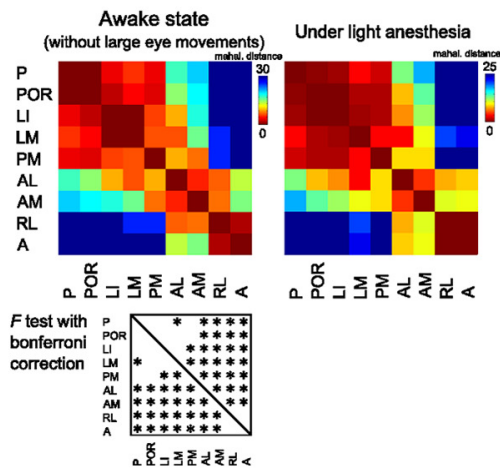

Figure 5. Spatiotemporal preferences of HVAs were consistent between awake and anesthetized mice. $A$, Experimental setup. B, Brief explanation of the pupil detection algorithm. We detected the pupil area by intensity threshold and computed the center of the detected pupil area by an ellipse fitting (see Materials and Methods). C, Representative traces of eye movements (top row, horizontal eye movements; middle row, vertical eye movements) and locomotion (bottom row). Although the eye movements were few in traces 1 and 2 (left and middle columns, respectively), large eye movements occurred in trace 3 (right column). We determined trials including large eye movements $\left(>10^{\circ}\right.$ ) as "trial with large eye movements" (see Materials and Methods). $\boldsymbol{D}$, Distributions of eye movements (top, horizontal eye movements; middle, vertical eye movements) and locomotion (bottom). Right, Frequencies of large eye movements ( $>10^{\circ}$ ) and locomotion. $E$, Peak points of the fitted response matrix in awake mice $(n=5$ mice). The spatiotemporal preferences were similar between the data of all trials and extracted trials without large eye movements. $\boldsymbol{F}$, Peak points of the fitted response matrix in anesthetized mice. The data are the same as those shown in Figure 3D. G, Colored matrices represent the Mahalanobis distances among HVAs ( $p<$ $0.0014, F$ test with Bonferroni correction). The functional segregation of HVAs in the awake state was very similar to that in the anesthetized state.

out of 36 in adults; Fig. $6 E, G)$. Moreover, response preferences were still variable across individual animals. Therefore, functional segregation of HVAs is still immature at P20 compared with results in adults.

At P25 ( $n=7$ mice; 5 matrices out of 70 were excluded), the variability of response preferences across individual animals was as low as that in adults (Fig. 6F). There were significant main effects among HVAs for both SPF and TF parameters (Fig. 6F, one-way ANOVA, SPF: $F_{(8,49)}=11.9, p<10^{-9}$; TF: $F_{(8,49)}=$ $\left.37.64, p<10^{-18}\right)$. As with adults, the preferred SPF in areas POR, $\mathrm{P}, \mathrm{PM}$, and $\mathrm{AM}$ were significantly higher than that in areas RL and A (Fig. $6 F, p<0.05$, post hoc Tukey's HSD test) and the preferred $\mathrm{TF}$ in areas RL and A were significantly higher than that in other areas (Fig. $6 F, p<0.05$, post hoc Tukey's HSD test). The number of significant differences in preferred SPF and TF was comparable to that in adults (Fig. $6 F, G$ ). Together, these results suggest that functional segregation of nine HVAs at P25 reaches an adult-like state.

Maturation of selectivity and functional segregation of HVAs Developmental analyses of the spatiotemporal selectivity in individual HVAs also supported gradual maturation of spatiotemporal selectivity. Figure 7, $A$ and $B$, shows the developmental change of preferred SPF and TF of V1 and All HVAs. Preferred SPF and TF were the peaks of fitted response matrices. In areas LM and AL, the preferred SPF showed a significant increase with age (Fig. $7 A$, one-way ANOVA, LM: $F_{(3,22)}=6.75, p<0.003 ; \mathrm{AL}: F_{(3,22)}=$ 4.07, $p<0.02$; post hoc Tukey's HSD test, $p<0.05)$. In V1 and areas $\mathrm{P}, \mathrm{POR}, \mathrm{LI}, \mathrm{PM}$, and $\mathrm{AM}$, there was a trend toward a gradual increase in preferred SPF with age, although this was not significant (Fig. 7A, one-way ANOVA, V1: $F_{(3,22)}=2.45, p=0.09$; P: $F_{(2,11)}=1.83, p=0.21 ;$ POR: $F_{(3,22)}=1.03, p=0.40 ; \mathrm{LI}: F_{(3,18)}=$ $2.7, p=0.08$; PM: $F_{(3,19)}=2.75, p=0.071 ; \mathrm{AM}: F_{(3,18)}=0.86$, $p=0.48)$. For TF preference, areas LM, PM, AL, RL, and A showed a trend toward gradual increase with age, although this was not significant (Fig. 7B, one-way ANOVA, LM: $F_{(3,19)}=0.9$, $p=0.46 ;$ PM: $F_{(3,22)}=1.5, p=0.24 ; \mathrm{AL}: F_{(3,22)}=1.5, p=0.24$; RL: $\left.F_{(3,22)}=2.2, p=0.12 ; \mathrm{A}: F_{(2,17)}=2.24, p=0.14\right)$. We also quantified the variance between individual animals in terms of the preferred SPF and TF (Fig. 7C,D). Although the SDs of preferred SPF and TF were not significantly different across ages, there was a trend for the SD to decrease, especially for TF (oneway ANOVA, SPF: $F_{(3,30)}=0.36, p=0.78$; TF: $F_{(3,30)}=1.75, p=$ 0.18 ). When we compared preferred SPF and TF between HVAs, the functional dissociation between HVAs became clearer with age (Fig. $7 E, F$ ) and we found that, as the mouse developed, in- 
A $\quad \operatorname{EO1}(\mathrm{n}=5$ mice $)$

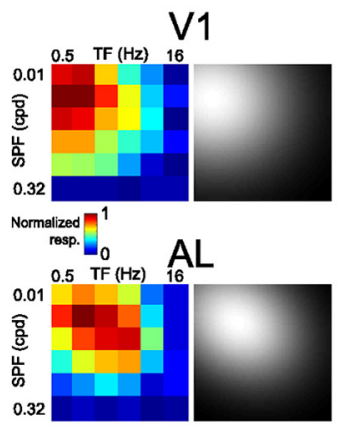

LM

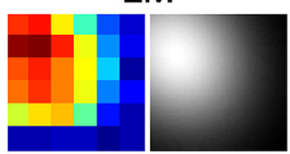

RL

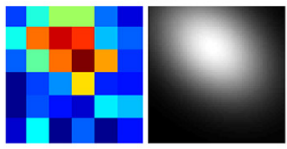

LI

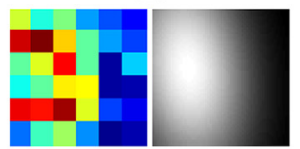

a

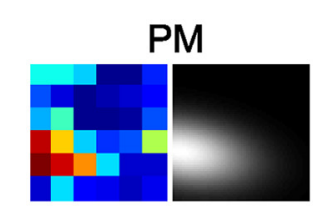

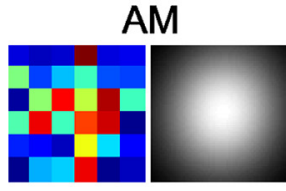

\section{B $\quad$ PD20 $(n=7$ mice $)$}
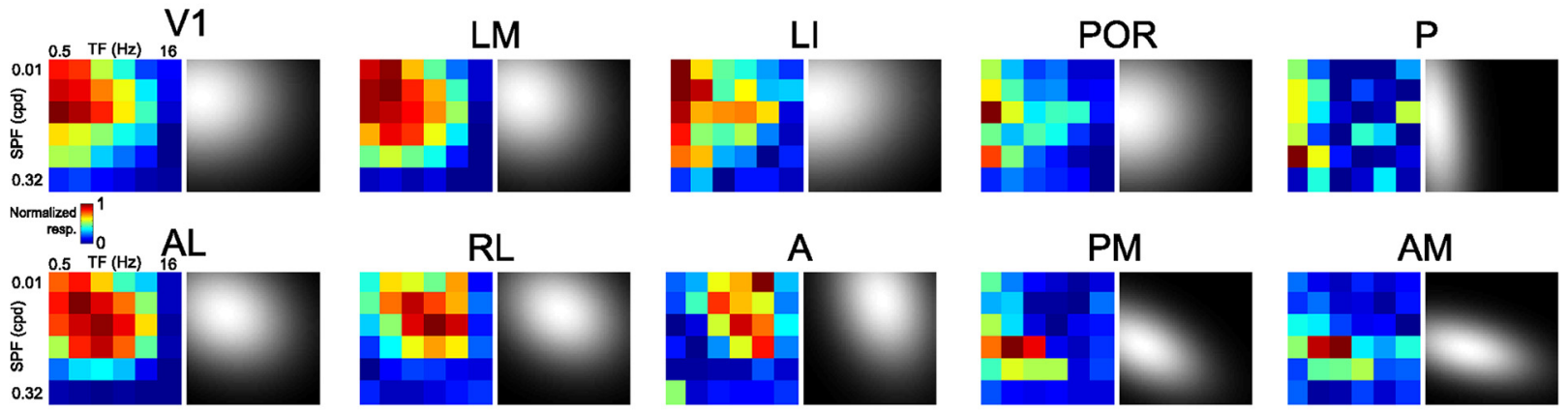

\section{C $\quad \operatorname{PD} 25(n=7$ mice $)$}
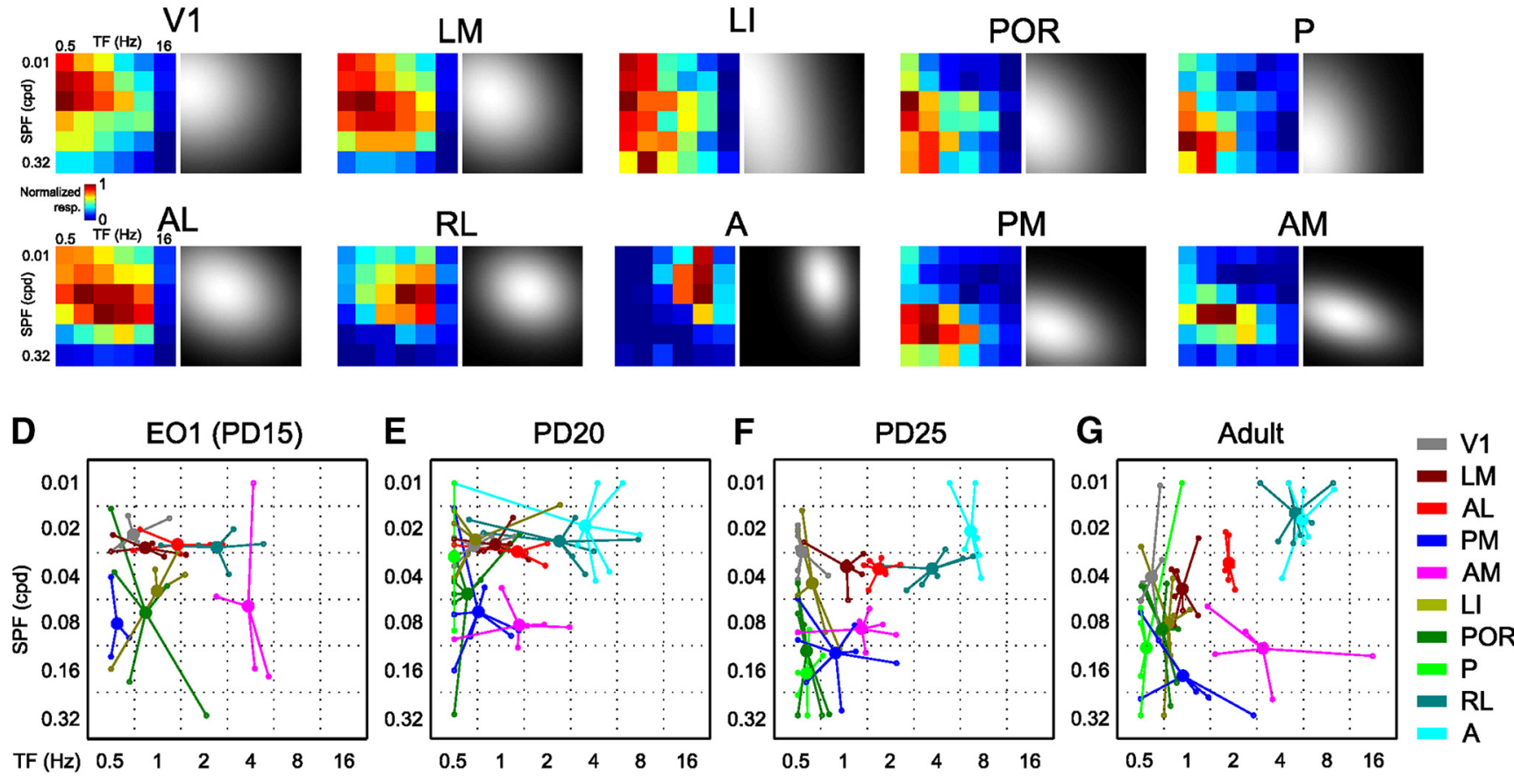

One-way ANOVA \& EO1 (PD15)

post hoc HSD test
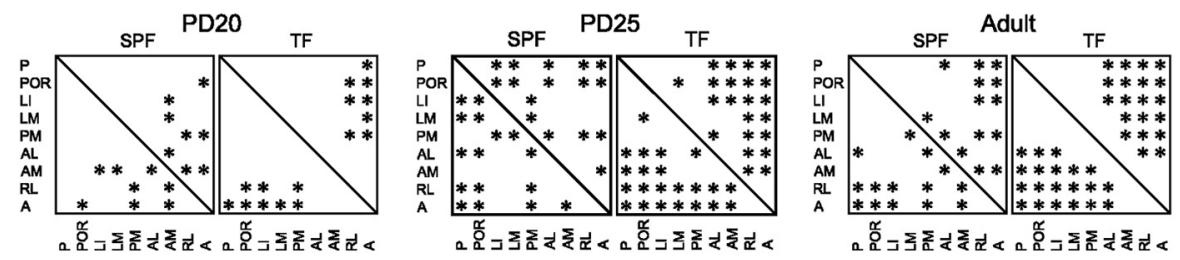

Figure 6. Spatiotemporal selectivity of developing HVAs at three time points. $\boldsymbol{A}-\boldsymbol{C}$, Response matrices of V1 and nine HVAs fitted with $2 D$ Gaussian fitting in developing mice at E01 ( $\boldsymbol{A})$, P2O (B), and P25 (C). The left color-coded matrix in each area is the averaged response matrices before the Gaussian fitting. $\boldsymbol{D}-\boldsymbol{G}$, Peak points of the fitted response matrices of developing mice at E01 (D), P20 (E), P25 (F), and adult mice $(\boldsymbol{G})$. Small open circles indicate the individual data. Large filled circles are averages of the open circles. The adult data $(\boldsymbol{G})$ are the same as those described in Figure 3D. Bottom, Results of one-way ANOVA and post hoc test. For SPF preference, there was no main effect at E01 (one-way ANOVA, $F_{(6,24)}=1.85, p=0.13$ ). At P20, (Figure legend continues.) 
creasing number of areal pairs showed significant difference (Fig. $7 E, F$, bottom). Therefore, these results corroborate the notion that the functional segregation of HVAs gradually maturate with age.

Finally, to examine the functional segregation of HVAs, we performed a discriminant analysis computing the inter-areal Mahalanobis distances of spatiotemporal selectivity among HVAs (Fig. 7G,H). The distances between ventral and dorsal HVAs (e.g., LI vs AM and POR vs RL) were short at EO1 and P20 and greatly increased at P25 (Fig. $7 G$ ). This result suggests that the functional segregation between the putative ventral and dorsal streams is obtained by P25. Similarly, the distances between HVAs in the putative dorsal stream (e.g., AL vs PM, AM vs RL, and PM vs RL) were also short at EO1 and P20 and increased at P25 (Fig. 7G), suggesting that the non-uniform functional properties of HVAs in the putative dorsal stream are established by P25. In all areal pairs, the distances at P25 and in adults were significantly larger than those at EO1 and P20 (Fig. $7 H$, one-way ANOVA, $F_{(3,125)}=9.88, p<10^{-5}$; post hoc Tukey's HSD test, $p<$ $0.05)$ and there was no significant difference between distances at P25 and in adults ( $p=0.26$, post hoc Tukey's HSD test). Together, these results suggest that the functional segregation of HVAs is immature at EO1 and P20 and quickly reaches the adultlike pattern by $\mathrm{P} 25$.

\section{Discussion}

\section{Functional and anatomical classification of mouse HVAs}

The nine HVAs are categorized into two groups based on their axonal projection patterns (Wang et al., 2012): a putative ventral stream that consists of lateral HVAs (LM, LI, POR, and P) and a putative dorsal stream that consists of anterior and medial HVAs (AL, PM, AM, RL, and A). Here, we performed functional categorization of nine HVAs, including three previously uncharacterized areas (i.e., POR, P, and A), based on spatiotemporal selectivity. Figure 8 shows the schematic of visual streams mainly based on previous anatomical studies (Wang et al., 2011, 2012) and the present study contributes a part of the schematic that describes which HVAs share similar spatiotemporal selectivity (third row in Fig. 8). In the putative ventral stream, area LM is considered as a gateway to this stream (Wang et al., 2011). Areas LI, POR, and P preferred gratings with high SPF and low TF (Fig. $3 D, E)$. Discriminant analysis revealed that areas LM, LI, POR, and $\mathrm{P}$ were categorized functionally into the same group and were separated from anterior HVAs (AL, AM, RL, and A) in the putative dorsal stream (Fig. $3 E$ ). These results indicate that functional classification of lateral HVAs (LM, LI, POR, and P) is consistent with their anatomical classification (Wang et al., 2012). A previous study reported, however, that axonal projection targets of HVAs in the putative ventral stream are similar but not uniform (Wang et al., 2012). Further classification of HVAs by complex functional profiling beyond spatiotemporal selectivity may enable the segregation of the ventral stream in accordance with the detailed anatomical profiles.

Unlike the agreement between functional and anatomical classification in the putative ventral stream, spatiotemporal selec-

\section{$\leftarrow$}

(Figure legend continued.) although there was a main effect on preferred SPF (one-way ANOVA, $F_{(8,46)}=5.27, p<0.0001$ ), the number of significant differences was less than that in adults. For TF preference, there were main effects at E01 and P20 (one-way ANOVA, E01: $F_{(6,24)}=7.2, p<0.0002, p<0.0004 ; P 20: F_{(8,46)}=6.45, p<1.32 \times 10^{-5}$ ), but the number of significant differences was less than that in adults. At P25, the number of significant differences in both preferred SPF and TF was comparable to that in adults (one-way ANOVA, SPF: $F_{(8,49)}=11.9, p<10^{-9} ; \mathrm{TF}: F_{(8,49)}=37.64, p<10^{-18} ;$ post hoc Tukey's HSD test, $\left.p<0.05\right)$. tivity of anterior/medial HVAs (AL, PM, AM, RL, and A) in the putative dorsal stream was not uniform and formed multiple functional groups (Fig. 3E). A recent study has demonstrated the functional distinctions between the anatomical ventral and dorsal HVAs without demonstrating the heterogeneity within the dorsal stream (Smith et al., 2017). The discrepancy between this study and ours may be attributed to a methodological difference: Smith et al. (2017) classified HVAs using across animal correlation of response magnitudes among HVAs, whereas the present study classified HVAs according to their spatiotemporal selectivity.

The present results suggest that the anterior/medial HVAs do not form a single stream despite massive reciprocal connections between them (Wang et al., 2012). Among the five HVAs, area AL is considered as the gateway because it receives the densest projection from V1 (Han et al., 2017) and in turn sends outputs to the remaining HVAs (Wang et al., 2011). Therefore, we placed area AL on the path to areas PM, AM, RL, and A in Figure 8. The three functionally distinct groups formed by the remaining four HVAs send their main anatomical projections to distinct target areas (Fig. 8) (Wang et al., 2012). Interestingly, each of these groups may have parallels with the primate dorsal substreams.

The first group, area PM, has strong connections to the retrosplenial (RS) and secondary cingulate areas (Cg2) (Wang et al., 2012). Like PM, area RS also prefers low-speed gratings with high SPF and low TF (Murakami et al., 2015) and encodes the memory of landmarks in the water maze task (Czajkowski et al., 2014). Furthermore, neurons in area RS are essential for the representation of head direction cells in the anterodorsal thalamic nucleus (Clark et al., 2010). Similarly, in primates, one substream in the dorsal stream runs through area RS and posterior cingulate cortex (Kravitz et al., 2011) and contributes to spatial navigation (Vann et al., 2009). The substream from area PM to RS/Cg2 may form a specialized substream for spatial navigation conserved across species (Fig. 8) (Vann et al., 2009; Yoder et al., 2011). Because area PM receives dense projection from both areas V1 and AL (Wang et al., 2011; Han et al., 2017), we placed area PM under these two areas in Figure 8.

The second group, area AM, sends strong projections to the motor and primary cingulate areas (Cg1) (Wang et al., 2012). Stimulation of the motor area and Cg1 evoke eye movements in rodents (Brecht et al., 2004). A recent study has reported that the part of the mouse parietal areas including area AM contributes to spatial memory-guided tasks (Harvey et al., 2012). In primates, visual areas, the frontal eye fields, and the lateral intraparietal areas form a specific substream in the dorsal pathway specialized for eye movements and spatial working memory (Kravitz et al., 2011). The substream from area AM to motor/Cg1 in mice may be homologous to this substream in primates.

Finally, the third group, consisting of areas RL and A, sends strong projections to the somatosensory and motor cortices. Area RL processes visuo-tactile information for multisensory integration (Olcese et al., 2013). Similarly, the parieto-premotor pathway in primates, a substream within the dorsal stream (Kravitz et al., 2011), contains not only motion-related visual features, but also coordinated maps of space and body position (Snyder et al., 1998; Sereno et al., 2006; Prevosto et al., 2010). The substream from areas RL and A to the somatomotor cortices in mice may be homologous to this substream in primates associated with multimodal visually guided action.

Despite having a smaller number of visual cortical areas, the network-level organization of the dorsal stream in mice may be analogous to that in primates (Kravitz et al., 2011). However, the 
A Preferred SPF $\square$ E01 $\square$ PD20 $\square$ PD25 Adult

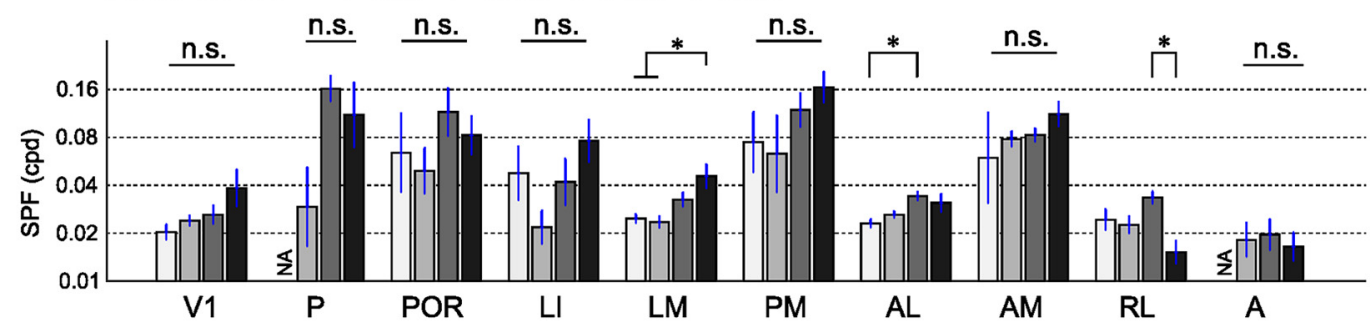

B Preferred TF $\square$ EO1 $\square$ PD20 $\square$ PD25 $\square$ Adult

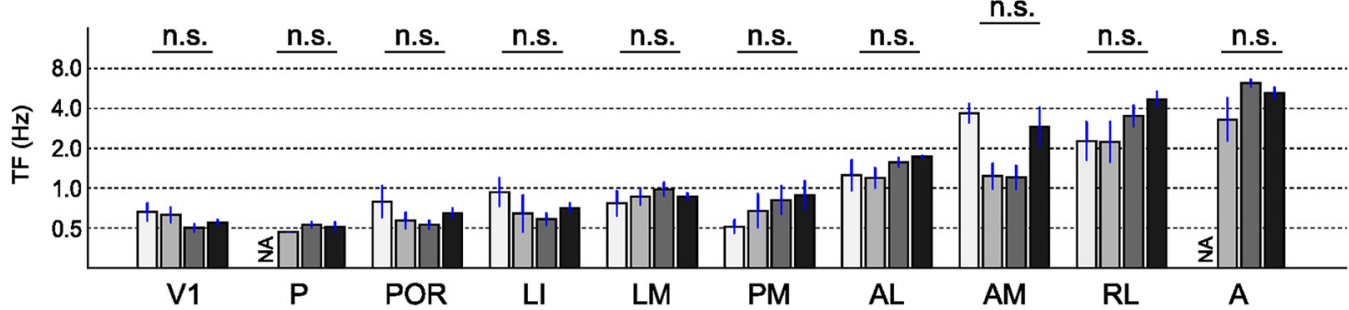

C STD (SPF)

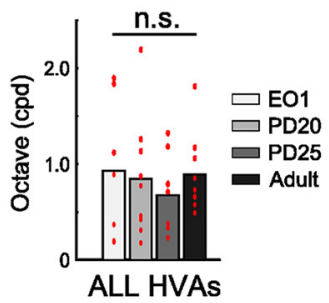

D STD (TF)

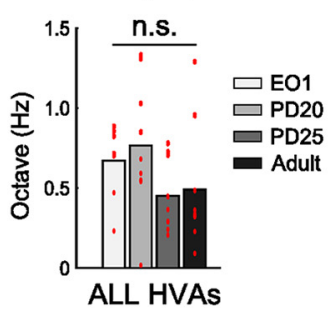

\section{$\mathbf{E}$ \\ Preferred SPF $\quad-\mathrm{LM}=\mathrm{PM}=\mathrm{LI}=\mathrm{P}=\mathrm{A}$ $-\mathrm{AL}=\mathrm{AM}=\mathrm{POR}=\mathrm{RL}$}

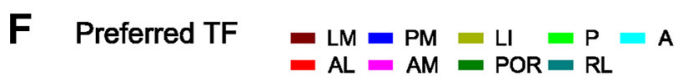

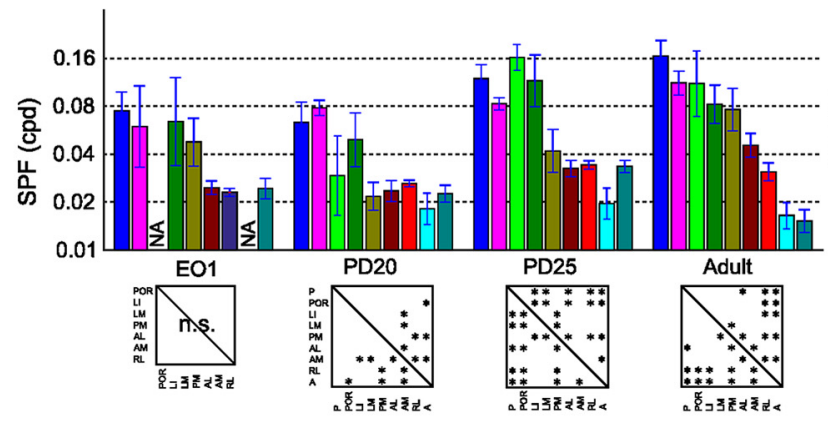

G Mahalanobis distance
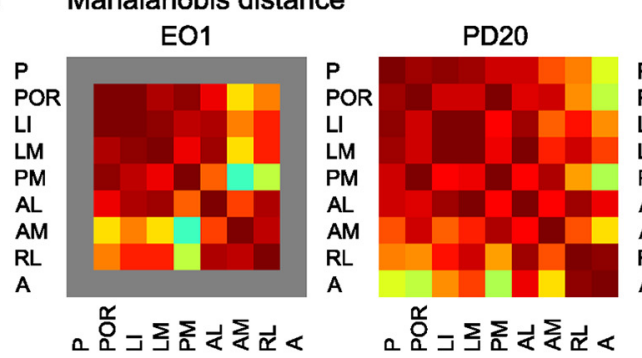

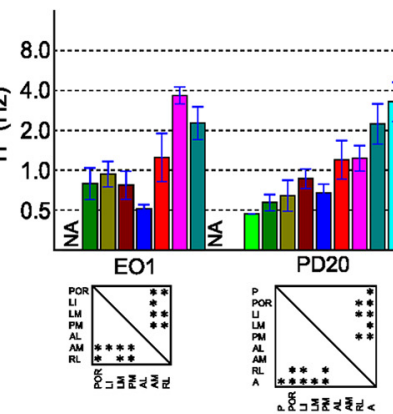

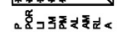
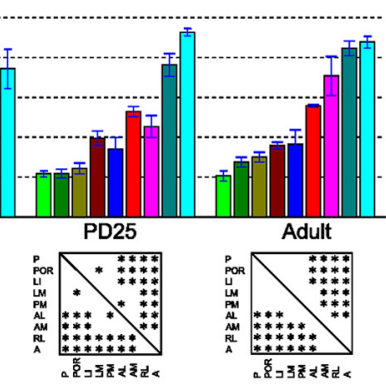

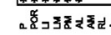

$\mathbf{H}$
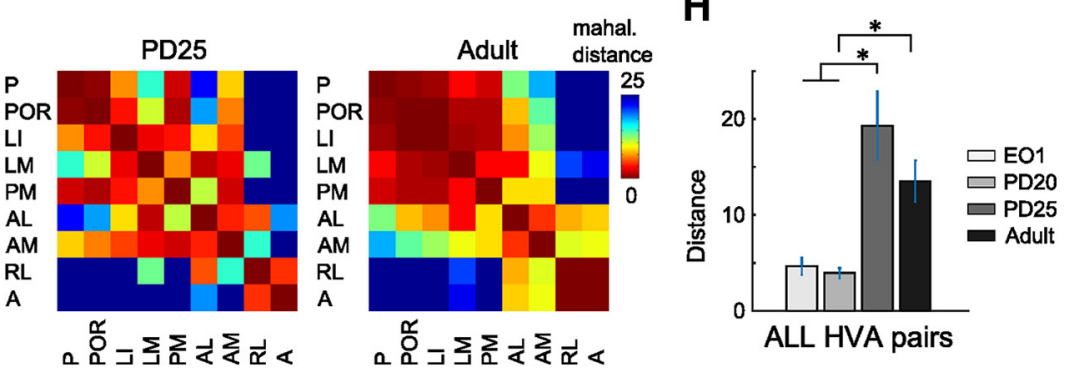

Figure 7. Comparison of spatiotemporal selectivity of HVAs between developing and adult mice. $\boldsymbol{A}$, Comparisons of preferred SPF between developing and adult mice in individual areas. Preferred SPF of areas $L M$ and AL increased significantly with age (one-way ANOVA, LM: $F_{(3,22)}=6.75, p<0.003 ; A L: F_{(3,22)}=4.07, p<0.02 ;$ posthoc Tukey's HSD test, $\left.p<0.05\right)$. B, Comparisons of the preferred TF between developing and adult mice in individual areas. $C, D, S D$ s of preferred SPF and TF. $E, F$, Comparison of preferred SPF and TF between HVAs. HVAs are listed in descending or ascending order of adult data in preferred SPF $(\boldsymbol{E})$ or preferred TF $(\boldsymbol{F})$, respectively. Bottom statistical panels are the same as those shown in Figure 6, D-G. $\boldsymbol{G}, \boldsymbol{H}$, Comparisons of inter-areal Mahalanobis distances among the four time points. The distances between HVAs were short at E01 and P20 and increased at P25 (G). In all HVA pairs, the inter-areal distances at E01 and P20 were significantly shorter than those in P25 and adult mice ( $\boldsymbol{H}$; one-way ANOVA, $F_{(3,125)}=9.88, p<10^{-5}$; post hoc Tukey's HSD test, $\left.p<0.05\right)$. Data are presented as mean \pm SEM.

correspondence between mice and primates is not perfect. Visual areas in the parieto-prefrontal pathway in primates, such as areas MT and LIP, generally prefer high-velocity stimuli (Cheng et al., 1994; Priebe et al., 2003; Miura et al., 2014), whereas area AM in mice prefers intermediate-velocity stimuli (Fig. 3D). Furthermore, the low SPF preference of areas RL and A may not be essential for visually guided action. Further exploration is necessary to clarify the correspondence between mice and primates.

Comparison with the reported spatiotemporal selectivity of HVAs

Previous studies have shown consistently that neurons in area AL prefer low SPF and high TF stimuli, whereas those in area PM prefer high SPF and low TF stimuli (Andermann et al., 2011; Marshel et al., 2011; Roth et al., 2012). Our results were consistent with these reports, although the TF preference in area AL in the present study was slightly lower than previously reported. In addition, we found that areas RL and AM respond strongly to high TF gratings, which was consistent with previous studies (Marshel et al., 2011; Tohmi et al., 2014).

Spatiotemporal selectivity in other HVAs is controversial. Some studies have reported that area LM has dorsal-stream-like properties: Neurons in area LM prefer low SPF and high TF stimuli (Marshel et al., 2011) and are selective to pattern direction (Juavinett and Callaway, 2015). However, other studies using 


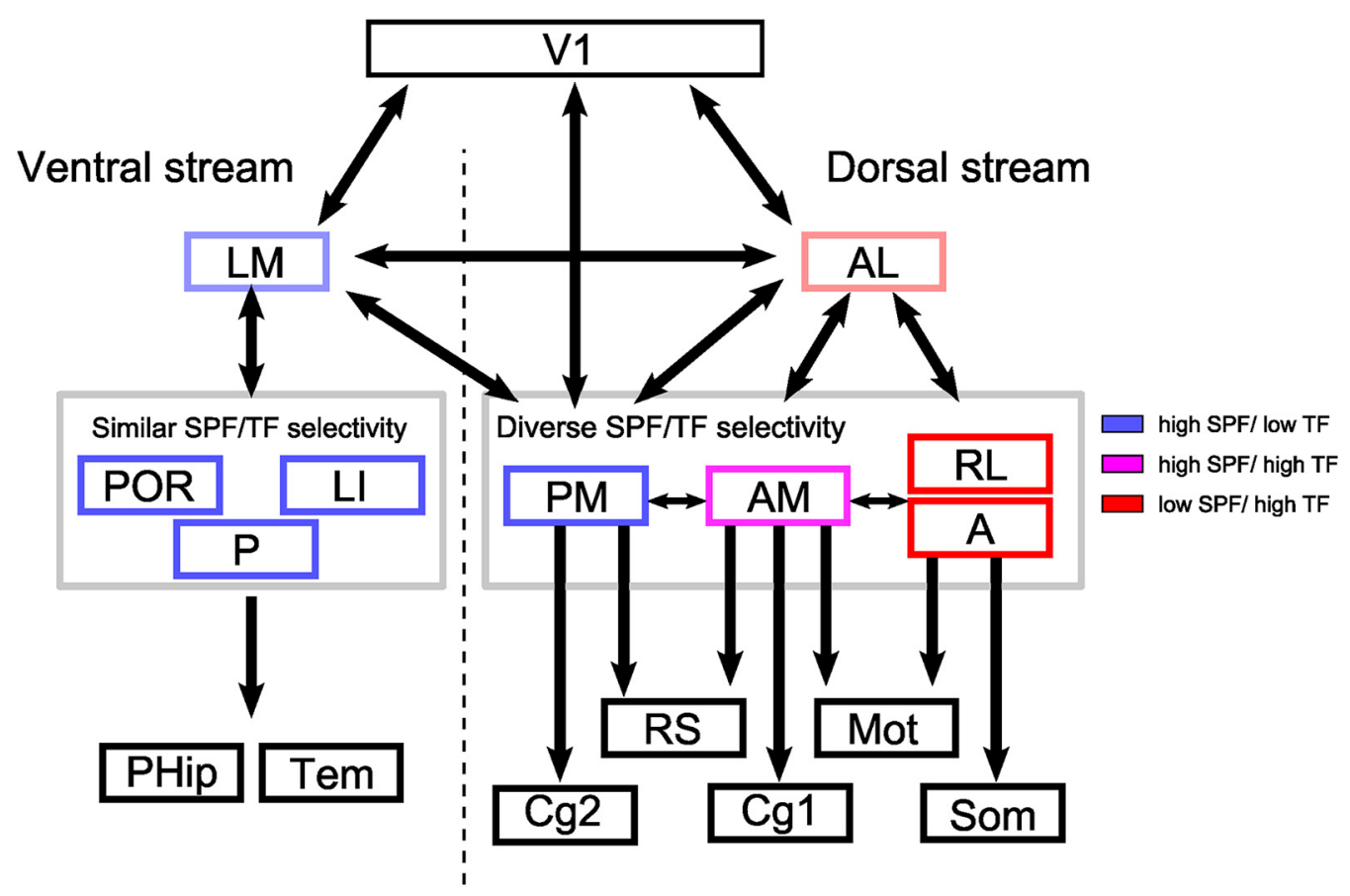

Figure 8. Summary of the anatomical (Wang et al., 2012) and functional streams of mouse HVAs. The schematic of visual streams is based on Wang et al. (2012). The putative ventral stream consists of four lateral HVAs (LM, LI, POR, and P) (Wang et al., 2012). Area LM is considered as a gateway to the putative ventral stream and areas LI, P, and POR send projections to parahippocampal (PHip) and temporal cortices (Tem) (Wang et al., 2011, 2012). These ventral HVAs share similar spatiotemporal selectivity (Fig. 3D,E) and this stream may process the pattern-related visual features for object recognition. The putative dorsal stream consists of anterior/medial HVAs (AL, PM, AM, RL, and A) (Wang et al., 2012). Area AL is considered to be a gateway to the putative dorsal stream (Wang et al., 2011). The current study indicates that the spatiotemporal selectivity of the remaining four HVAs is not uniform and these areas form three functionally distinct groups (Fig. 3D,E) that have different anatomical targets (Wang et al., 2012). The first group, area PM, has strong connections to the RS and Cg2 areas. The second group, area AM, sends strong projections to the motor (Mot) and $\mathrm{Cg} 1$ areas. Finally, the third group, areas RL and A, sends strong projections to the somatosensory (Som) and motor cortices.

two-photon imaging of the axonal projections from V1 to area LM have reported that area LM has spatiotemporal selectivity similar to that of V1 (Glickfeld et al., 2013; Matsui and Ohki, 2013). The present study was consistent with the latter studies. Similarly, although it has been reported that neurons in area LI prefer high SPF and high TF stimuli (Marshel et al., 2011), in our results, area LI preferred high SPF and low TF stimuli. Some of the discrepancies between studies may be attributed to differences in the precise stimulus parameters used to evaluate spatiotemporal selectivity. In some studies (Marshel et al., 2011; Matsui and Ohki, 2013), the TF or SPF was fixed in one parameter for the evaluation of the SPF or TF preference, respectively. Other studies, including the present one, used a set of 36 gratings composed of combinations of six SPF and six TF (Andermann et al., 2011; Glickfeld et al., 2013). It is also important to consider the potential sampling bias in two-photon imaging. Wide-field imaging obtains visual responses averaged across all excitatory neurons in each HVA, whereas two-photon imaging monitors activities of a relatively small number of neurons in a limited field of view. If there was functional heterogeneity of neurons in area LM, then the small number of sampled neurons in two-photon imaging could result in sampling bias.

Development of spatiotemporal selectivity of HVAs after EO Recently, some visual functions of HVAs, response magnitude, orientation selectivity, and receptive field, have been reported to develop gradually after EO (Smith et al., 2017). In addition to these functions, the present study revealed that functional segregation of HVAs was also immature soon after EO (Fig. 6) and gradually developed with age (Fig. 7). Consistent with the present results, SPF selectivity in mouse V1 gradually matures over $7 \mathrm{~d}$ after EO (Hoy and Niell, 2015). During the same period, we found that HVAs were segregated functionally gradually into an adult-like pattern (Fig. 7). These developmental profiles of V1 and HVAs in mice are similar to those in cats and monkeys, in which V1 and the secondary visual area show gradual maturation of both SPF and TF selectivity after birth (DeAngelis et al., 1993; Chino et al., 1997; Zheng et al., 2007).

When we compared preferred SPF and TF in individual areas, the developmental change of preferred SPF and TF seemed small (Fig. $7 A, B$ ). However, when the difference between areal pairs were considered, we found that, as the mouse developed, increasing number of areal pairs showed significant difference (Fig. $7 E, F)$. In addition, the comparison of Mahalanobis distances among HVAs, which took into account both of the developmental changes of preferred SPF and TF, showed significant development of functional dissociation among HVAs (Fig. 7G,H). Therefore, we think that, although the developmental change within individual areas seems small, development of functional segregation between areas is significant. Furthermore, the variance of preferred SPF and that of TF between individual mice both had a trend to decrease slightly, suggesting that it is likely that both the functional convergence between individual animals and the developmental change of preferred SPF and TF contribute to the development of functional segregation between HVAs.

What is the developmental mechanism of functional segregation among HVAs? One possibility is a change in axonal projections from V1. Previous studies have found that each HVA receives distinct visual information from V1 (Glickfeld et al., 2013; Matsui and Ohki, 2013). Immature functional segregation of HVAs soon after EO may be due to nonspecific projections from V1 to HVAs. Maturation of functional segregation after EO 
may follow refinement of V1 axonal projections to send specific visual features to each HVA. Another possibility is a change of inputs from the extrageniculate pathway, which runs from the retina via the superior colliculus and lateral posterior nucleus of the thalamus to HVAs (Gale and Murphy, 2014; Tohmi et al., 2014). Immature functional segregation among HVAs soon after EO may be due to immature extrageniculate pathway. It will be of great interest to test these possibilities using two-photon imaging of axonal activity throughout development.

\section{References}

Akerboom J et al. (2012) Optimization of a GCaMP calcium indicator for neural activity imaging. J Neurosci 32:13819-13840. CrossRef Medline

Andermann ML, Kerlin AM, Roumis DK, Glickfeld LL, Reid RC (2011) Functional specialization of mouse higher visual cortical areas. Neuron 72:1025-1039. CrossRef Medline

Brecht M, Krauss A, Muhammad S, Sinai-Esfahani L, Bellanca S, Margrie TW (2004) Organization of rat vibrissa motor cortex and adjacent areas according to cytoarchitectonics, microstimulation, and intracellular stimulation of identified cells. J Comp Neurol 479:360-373. CrossRef Medline

Chen TW, Wardill TJ, Sun Y, Pulver SR, Renninger SL, Baohan A, Schreiter ER, Kerr RA, Orger MB, Jayaraman V, Looger LL, Svoboda K, Kim DS (2013) Ultrasensitive fluorescent proteins for imaging neuronal activity. Nature 499:295-300. CrossRef Medline

Cheng K, Hasegawa T, Saleem KS, Tanaka K (1994) Comparison of neuronal selectivity for stimulus speed, length, and contrast in the prestriate visual cortical areas V4 and MT of the macaque monkey. J Neurophysiol 71: 2269-2280. CrossRef Medline

Chino YM, Smith EL 3rd, Hatta S, Cheng H (1997) Postnatal development of binocular disparity sensitivity in neurons of the primate visual cortex. J Neurosci 17:296-307. Medline

Clark BJ, Bassett JP, Wang SS, Taube JS (2010) Impaired head direction cell representation in the anterodorsal thalamus after lesions of the retrosplenial cortex. J Neurosci 30:5289-5302. CrossRef Medline

Czajkowski R, Jayaprakash B, Wiltgen B, Rogerson T, Guzman-Karlsson MC, Barth AL, Trachtenberg JT, Silva AJ (2014) Encoding and storage of spatial information in the retrosplenial cortex. Proc Natl Acad Sci U S A 111:8661-8666. CrossRef Medline

DeAngelis GC, Ohzawa I, Freeman RD (1993) Spatiotemporal organization of simple-cell receptive fields in the cat's striate cortex. I. general characteristics and postnatal development. J Neurophysiol 69:1091-1117. Medline

Desimone R, Schein SJ (1987) Visual properties of neurons in area V4 of the macaque: sensitivity to stimulus form. J Neurophysiol 57:835-868. Medline

Felleman DJ, Van Essen DC (1991) Distributed hierarchical processing in the primate cerebral cortex. Cereb Cortex 1:1-47. Medline

Gale SD, Murphy GJ (2014) Distinct representation and distribution of visual information by specific cell types in mouse superficial superior colliculus. J Neurosci 34:13458-13471. CrossRef Medline

Garrett ME, Nauhaus I, Marshel JH, Callaway EM (2014) Topography and areal organization of mouse visual cortex. J Neurosci 34:12587-12600. CrossRef Medline

Glickfeld LL, Andermann ML, Bonin V, Reid RC (2013) Cortico-cortical projections in mouse visual cortex are functionally target specific. Nat Neurosci 16:219-226. CrossRef Medline

Glickfeld LL, Reid RC, Andermann ML (2014) A mouse model of higher visual cortical function. Curr Opin Neurobiol 24:28-33. CrossRef Medline

Gorski JA, Talley T, Qiu M, Puelles L, Rubenstein JL, Jones KR (2002) Cortical excitatory neurons and glia, but not GABAergic neurons, are produced in the Emx1-expressing lineage. J Neurosci 22:6309-6314. Medline

Han Y, Kebschull JM, Campbell RAA, Cowan D, Imhof F, Zador AM, MrsicFlogel TD (2017) A single-cell anatomical blueprint for intracortical information transfer from primary visual cortex. Biorxiv. Available at http://www.biorxiv.org/content/early/2017/06/09/148031.

Harvey CD, Coen P, Tank DW (2012) Choice-specific sequences in parietal cortex during a virtual-navigation decision task. Nature 484:62-68. CrossRef Medline

Hoy JL, Niell CM (2015) Layer-specific refinement of visual cortex function after eye opening in the awake mouse. J Neurosci 35:3370-3383. CrossRef Medline
Juavinett AL, Callaway EM (2015) Pattern and component motion responses in mouse visual cortical areas. Curr Biol 25:1759-1764. CrossRef Medline

Kravitz DJ, Saleem KS, Baker CI, Mishkin M (2011) A new neural framework for visuospatial processing. Nat Rev Neurosci 12:217-230. CrossRef Medline

Kravitz DJ, Saleem KS, Baker CI, Ungerleider LG, Mishkin M (2013) The ventral visual pathway: An expanded neural framework for the processing of object quality. Trends Cogn Sci 17:26-49. CrossRef Medline

Lewis JW, Van Essen DC (2000) Corticocortical connections of visual, sensorimotor, and multimodal processing areas in the parietal lobe of the macaque monkey. J Comp Neurol 428:112-137. CrossRef Medline

Madisen L et al. (2015) Transgenic mice for intersectional targeting of neural sensors and effectors with high specificity and performance. Neuron 85:942-958. CrossRef Medline

Markov NT, Vezoli J, Chameau P, Falchier A, Quilodran R, Huissoud C, Lamy C, Misery P, Giroud P, Ullman S, Barone P, Dehay C, Knoblauch K, Kennedy H (2014) Anatomy of hierarchy: feedforward and feedback pathways in macaque visual cortex. J Comp Neurol 522:225-259. CrossRef Medline

Marshel JH, Garrett ME, Nauhaus I, Callaway EM (2011) Functional specialization of seven mouse visual cortical areas. Neuron 72:1040-1054. CrossRef Medline

Matsui T, Ohki K (2013) Target dependence of orientation and direction selectivity of corticocortical projection neurons in the mouse V1. Front Neural Circuits 7:143. CrossRef Medline

Matsui T, Murakami T, Ohki K (2016) Transient neuronal coactivations embedded in globally propagating waves underlie resting-state functional connectivity. Proc Natl Acad Sci U S A 113:6556-6561. CrossRef Medline

Maunsell JH, van Essen DC (1983) The connections of the middle temporal visual area (MT) and their relationship to a cortical hierarchy in the macaque monkey. J Neurosci 3:2563-2586. Medline

Miura K, Inaba N, Aoki Y, Kawano K (2014) Difference in visual motion representation between cortical areas MT and MST during ocular following responses. J Neurosci 34:2160-2168. CrossRef Medline

Murakami T, Yoshida T, Matsui T, Ohki K (2015) Wide-field ca(2+) imaging reveals visually evoked activity in the retrosplenial area. Front Mol Neurosci 8:20. CrossRef Medline

Nassi JJ, Callaway EM (2009) Parallel processing strategies of the primate visual system. Nat Rev Neurosci 10:360-372. CrossRef Medline

Olcese U, Iurilli G, Medini P (2013) Cellular and synaptic architecture of multisensory integration in the mouse neocortex. Neuron 79:579-593. CrossRef Medline

Peirce JW (2007) PsychoPy: psychophysics software in python. J Neurosci Methods 162:8-13. CrossRef Medline

Prevosto V, Graf W, Ugolini G (2010) Cerebellar inputs to intraparietal cortex areas LIP and MIP: Functional frameworks for adaptive control of eye movements, reaching, and arm/eye/head movement coordination. Cereb Cortex 20:214-228. CrossRef Medline

Priebe NJ, Cassanello CR, Lisberger SG (2003) The neural representation of speed in macaque area MT/V5. J Neurosci 23:5650-5661. Medline

Rizzolatti G, Matelli M (2003) Two different streams form the dorsal visual system: Anatomy and functions. Exp Brain Res 153:146-157. CrossRef Medline

Roth MM, Helmchen F, Kampa BM (2012) Distinct functional properties of primary and posteromedial visual area of mouse neocortex. J Neurosci 32:9716-9726. CrossRef Medline

Saleem AB, Ayaz A, Jeffery KJ, Harris KD, Carandini M (2013) Integration of visual motion and locomotion in mouse visual cortex. Nat Neurosci 16:1864-1869. CrossRef Medline

Sereno MI, Huang RS (2006) A human parietal face area contains aligned head-centered visual and tactile maps. Nat Neurosci 9:1337-1343. CrossRef Medline

Smith IT, Townsend LB, Huh R, Zhu H, Smith SL (2017) Streamdependent development of higher visual cortical areas. Nat Neurosci 20: 200-208. CrossRef Medline

Smith SL, Häusser M (2010) Parallel processing of visual space by neighboring neurons in mouse visual cortex. Nat Neurosci 13:1144-1149. CrossRef Medline

Snyder LH, Grieve KL, Brotchie P, Andersen RA (1998) Separate body- and world-referenced representations of visual space in parietal cortex. Nature 394:887-891. CrossRef Medline 
Tian L, Hires SA, Mao T, Huber D, Chiappe ME, Chalasani SH, Petreanu L, Akerboom J, McKinney SA, Schreiter ER, Bargmann CI, Jayaraman V, Svoboda K, Looger LL (2009) Imaging neural activity in worms, flies and mice with improved GCaMP calcium indicators. Nat Methods 6:875881. CrossRef Medline

Tohmi M, Meguro R, Tsukano H, Hishida R, Shibuki K (2014) The extrageniculate visual pathway generates distinct response properties in the higher visual areas of mice. Curr Biol 24:587-597. CrossRef Medline

Ungerleider LG, Mishkin M (1982) Two cortical systems. In: Analysis of visual behavior (Ingle DJ, Goodale MA, Mansfield RJW, eds), pp 549586. Cambridge, MA: Massachusetts Institute of Technology.

Vann SD, Aggleton JP, Maguire EA (2009) What does the retrosplenial cortex do? Nat Rev Neurosci 10:792-802. CrossRef Medline

Wang Q, Burkhalter A (2007) Area map of mouse visual cortex. J Comp Neurol 502:339-357. CrossRef Medline
Wang Q, Gao E, Burkhalter A (2011) Gateways of ventral and dorsal streams in mouse visual cortex. J Neurosci 31:1905-1918. CrossRef Medline

Wang Q, Sporns O, Burkhalter A (2012) Network analysis of corticocortical connections reveals ventral and dorsal processing streams in mouse visual cortex. J Neurosci 32:4386-4399. CrossRef Medline

Yoder RM, Clark BJ, Taube JS (2011) Origins of landmark encoding in the brain. Trends Neurosci 34:561-571. CrossRef Medline

Zariwala HA, Borghuis BG, Hoogland TM, Madisen L, Tian L, De Zeeuw CI, Zeng H, Looger LL, Svoboda K, Chen TW (2012) A cre-dependent GCaMP3 reporter mouse for neuronal imaging in vivo. J Neurosci 32: 3131-3141. CrossRef Medline

Zheng J, Zhang B, Bi H, Maruko I, Watanabe I, Nakatsuka C, Smith EL3rd, Chino YM (2007) Development of temporal response properties and contrast sensitivity of V1 and V2 neurons in macaque monkeys. J Neurophysiol 97:3905-3916. CrossRef Medline 